Référence de l'article :

Gepner, B. (2006). Constellation autistique, mouvement, temps et pensée. Malvoyance de l'ÉMotion, autres désordres du traitement temporospatial des flux sensoriels et dyssynchronie dans l'autisme. Devenir, 18, 4, 333-379.

\title{
Constellation autistique, mouvement, temps et pensée*
}

\author{
Malvoyance de l'É-Motion, \\ autres désordres du traitement temporospatial des flux sensoriels \\ et dyssynchronie dans l'autisme
}

\section{Bruno Gepner}

Praticien Hospitalier, Centre Hospitalier Montperrin et Chercheur associé, Laboratoire Parole et Langage, UMR CNRS 6057, Université de Provence, Aix-en-Provence

Adresse de l'auteur :

Dr Bruno Gepner, Service de Psychiatrie infanto-juvénile (13I08), Centre Hospitalier Montperrin. 109, Avenue du Petit Barthélémy, 13617 Aix-en-Provence.

E-mail : bruno.gepner@Ipl.univ-aix.fr

* Cet article invité constitue une nouvelle synthèse des travaux de Bruno Gepner et de ses collaborateurs. Il prolonge l'article «Malvoyance du mouvement dans l'autisme ? Une nouvelle approche neuropsychopathologique développementale», paru en 2001 dans $L a$ Psychiatrie de l'Enfant. 


\section{Résumé}

Dans cet article, nous poursuivons notre triple approche clinique, théorique et expérimentale des désordres de la constellation autistique, appelée Malvoyance de l'É-Motion et autres désordres $d u$ traitement temporospatial des flux sensoriels. Selon cette approche, les personnes atteintes de désordres autistiques présentent un degré plus ou moins important de difficultés dans la perception et l'intégration des mouvements physiques et biologiques, et plus généralement dans la perception en ligne des événements ou flux sensoriels en provenance du monde environnemental, ainsi que dans le couplage sensorimoteur et la production en temps réel d'événements moteurs et de communication verbale et émotionnelle, et ce dès les débuts de leur vie post-natale. En d'autres termes, le monde environnant va trop vite et change trop rapidement pour certains enfants autistes. Leurs perceptions des différents événements ou flux sensoriels se désynchronisent les unes des autres. Au lieu de se faire de manière unifiée et synchronisée, l'intégration et l'inscription du mouvement, de la durée et du temps dans le corps et la pensée de l'enfant autiste se font de manière fragmentée et désynchronisée. Vivant dans un espace-temps différent du nôtre, l'enfant autiste ne peut dès lors se lier ni s'accorder de manière adéquate avec le monde physique et humain, ni relier le monde externe avec son monde interne. Certains enfants autistes parviennent à corriger ou compenser en partie leur perception discontinue, non unifiée et incohérente du monde environnemental, en le ralentissant, en le figeant, en le répétant et/ou en surinvestissant des singularités ou des détails d'événements sensoriels, voire en collant adhésivement à l'objet. Nous montrerons comment notre approche permet d'ouvrir pour les personnes autistes des perspectives novatrices et prometteuses concrètes dans le domaine de la rééducation de leur communication verbale et émotionnelle par le biais d'un logiciel de ralentissement synchronisé des mouvements faciaux et de la parole des partenaires interactifs.

Sur le plan neurobio-physiologique, il est vraisemblable que ces désordres d'intégration temporo-spatiale soient sous-tendus par une dyssynchronie neuronale et une dysconnectivité fonctionnelle multi-système (c'est-à-dire impliquant de nombreux systèmes neurofonctionnels interconnectés). Les désordres autistiques, en tant qu'exemple particulier d'états de conscience, permettent d'éclairer le fonctionnement et les dysfonctionnements du psychismecerveau humain.

Mots-clés : autisme, émotion, mouvement, temps, connectivité neuronale, synchronisation neuronale, conscience 


\title{
Autistic constellation, movement, time and thought. E-Motion mis-sight, other temporospatial processing disorders of sensory flows and dyssynchrony in autism
}

\begin{abstract}
In this article, we pursue our threefold approach -clinical, theoretical and experimental- of the autistic constellation's disorders, called E-Motion mis-sight and other temporospatial processing disorders of sensory flows. According to this approach, persons with autistic spectrum disorders present more or less difficulties in the perception and integration of physical and biological movements, and more generally in perceiving on line sensory events and sensory flows coming from the environmental world, as well as in sensorymotor coupling and production on time of motor and verbal and emotional communication events. These difficulties occur from the very beginning of their life. In other words, the environmental world is going and changing too fast for at least some autistic persons. Their perception of various sensory flows is therefore desynchronized. Instead of being unified, coherent, continuous and synchronized, integration and inscription of motion, time and duration in their mind and body appear to be spatially fragmented and temporally desynchronized. Living in a different space-time, autistic children cannot connect nor tune with physical and human world adequately, neither can they connect their inner world with external world properly. Some autistic children succeed in correcting or compensating more or less their fragmented, discontinuous and desynchronized perception of their environment, by slowing down it, stopping it, repeting it, and/or by over-self-involving perceptually in details of sensory events, and even by sticking adhesively to objects. We willll show how our approach is able to open new promissing perspectives for reeducation of verbal and emotional communication disorders of autistic persons via an original software aimed at slowing down simultaneously facial movements and speech of their care takers.

At the neurofunctional level, these temporospatial processing disorders may be very plausibly accounted by a multi-system neural dyssynchronization and dysconnectivity, involving numerous interconnected neurofunctional systems. In conclusion, autistic disorders, as a particular case of conscious state, shed light on the functioning and disfunctioning of human brain-psychism.
\end{abstract}

\section{Key-words}

Autism, emotion, movement, time, neural connectivity, neural synchronization, consciousness 


\section{Introduction}

L'auteur de science fiction américain, Philip K. Dick, a écrit dans son livre Martian time-sleep (1964) :

"(...) - Il y a une nouvelle théorie concernant l'autisme, déclara le Dr glaub. Qui nous vient de Berghölzlei, en Suisse. Je voulais en discuter avec vous car elle semble nous offrir de nouvelles perspectives dans le cas de votre fils.

- J'en doute, répondit Steiner. Le Dr Glaub ne parut pas l'entendre, et continua.

- Elle suppose un trouble dans la perception de la durée chez l'individu autistique, de sorte que son environnement est tellement accéléré qu'il ne peut plus l'affronter; en fait, il est incapable de le percevoir correctement, exactement comme si nous regardions un programme de télévision accéléré, dans lequel les objets fileraient si vite qu'ils en deviendraient invisibles, et dont le son ne serait plus qu'un charabia incompréhensible... rien qu'un baragouinage très aigu. En suivant cette nouvelle théorie, on pourrait placer l'enfant autistique dans une chambre close avec un écran sur lequel on lui pojetterait au ralenti des séquences filmées. Le son et l'image seraient ralentis à une vitesse tellement lente que ni vous ni moi ne pourrions percevoir le moindre mouvement, ni reconnaître le bruit comme des paroles humaines.

- Fascinant, dit Steiner d'une voix lasse (...) ».

La réalité dépasse parfois la fiction. Parfois la réalité reste en deça de la fiction, mais pour combien de temps? La fiction précède souvent la réalité, et peut même l'induire. Parfois, ce peut être l'inverse. Dans le roman de Philip K. Dick, la planète Mars est une banlieue de la Terre, et les enfants autistes sont parqués dans un camp de concentration sur la planète rouge : sombre fiction. Et Steiner, le père d'un enfant autiste interné dans ce camp, est désespéré et finit par se suicider : fiction tragique ... Parfois, la fiction colle à la réalité.

Le but de cet article est d'essayer de montrer qu'un certain nombre de personnes présentant des désordres de la constellation autistique souffrent d'une distortion de leur perception du monde environnemental physique et humain. Plus précisément, le monde environnemental irait trop vite pour certaines personnes autistes. Ainsi, l'idée d'un trouble de la perception du temps et de la durée dans l'autisme, née dans l'imagination de Philip K. Dick il y a un peu plus de 40 ans, a sans doute quelque chose à voir avec le vécu perceptif quotidien de certaines personnes autistes. Nous allons en effet exposer différents arguments autobiographiques, cliniques et expérimentaux montrant qu'au moins certains enfants, adolescents et adultes avec autisme souffrent de désordres du traitement temporospatial des événements sensoriels dans les modalités visuelles, sonores et/ou tactilo-kinesthésiques. Nous essaierons également de montrer que notre approche neuropsychologique développementale présente un avantage par rapport aux autres théories contemporaines de l'autisme, en ce qu'elle pourrait ouvrir, dans un avenir relativement proche, de nouvelles perspectives pour la rééducation des troubles perceptifs et sensorimoteurs et des désordres de la communication verbale et émotionnelle des personnes autistes. Nous proposerons aussi de considérer ce que pourraient être les corrélats neurobiologiques de ces troubles du traitement temporospatial des événements multisensoriels, à la lumière des concepts actuels de synchronisation et de connectivité neuronale, et développerons l'hypothèse de dyssynchronie et dysconnectivité multisystème dans l'autisme. Nous envisagerons enfin des développements psychologiques et philosophiques de notre approche dans une perspective dualiste-interactionniste des relations psychisme-cerveau.

Mais avant cela, nous souhaitons rappeler quelques points de vue généraux sur l'autisme et le traitement des personnes autistes. Le syndrome d'autisme infantile a été décrit pour la première fois par Kanner (1943). Malgré des progrès substantiels depuis 60 ans dans le diagnostic, le traitement, et l'accompagnement des enfants, adolescents et adultes souffrant d'autisme et de troubles apparentés en France, les familles nous rappellent quotidiennement par leurs demandes, leurs souffrances, et leurs errances, que de graves lacunes persistent dans 
tous ces domaines tant du point de vue qualitatif que quantitatif. Depuis 10 ans, les pouvoirs publics et les organismes de tutelle font un effort en direction des personnes autistes et de leurs familles, effort notable mais qui reste encore très insuffisant. En toile de fond, il semble évident que les insuffisances dans le traitement de l'autisme sont essentiellement dues à un manque persistant de compréhension des mécanismes neuro-bio-physio-psychopathogéniques qui sous-tendent les désordres du spectre autistiques (Tardif \& Gepner, 2003). Ce manque de compréhension globale a produit et continue de produire des développements théoricocliniques et des applications thérapeutiques très divers et parfois hasardeux et/ou incompatibles.

Comme nous l'avons déjà souligné ailleurs (Gepner, 2005), l'énigme de l'autisme elle-même persiste pour plusieurs raisons, dont certaines sont brièvement résumées ici : 1) il n'y a pas UN autisme, mais une constellation de troubles neurodéveloppementaux complexes répertoriés sous la rubrique des troubles envahissants du développement (APA, 1994 ; WHO, 1992), ou des désordres du spectre de l'autisme (Rapin, 2002), situés le long d'un continuum de l'autisme (Grandin, 1995) ; 2) Il n'y a pas UNE cause de l'autisme, mais une multiplicité de facteurs de risque génétiques (plusieurs gènes de neurodéveloppement impliqués, en cours d'identification), épigénétiques et environnementaux (pré-, péri- ou post-nataux) en proportion variable, affectant le développement épigénétique du cerveau (Changeux, 1983) et l'auto-organisation cérébrale et psychique des personnes autistes (Bourguignon, 1981; Gepner \& Soulayrol, 1994) ; 3) Entre ces facteurs de risque affectant le développement du système nerveux central et leurs conséquences sur tel individu, il existe de très nombreux mécanismes physiopathogéniques complexes impliquant différents niveaux d'organisation, de complexité et d'intégration croissantes (neurobiologique, neurophysiologique, neuropsychologique), et affectant de nombreux systèmes neurofonctionnels, territoires et voies neurologiques enchevêtrés et interconnectés (Waterhouse et al., 1996 ; Gepner, 2001) ; 4) Il existe également des réactions émotionnelles et psychologiques variées (dépression, anxiété, panique, colère, inhibition, détresse...) qui influencent et colorent le caractère, la personnalité et les comportements de telle personne avec autisme dans telle ou telle voie ; 5) Finalement, et pour compliquer encore davantage le tableau, il existe plusieurs désordres neurodéveloppementaux comorbides, associés aux syndromes autistiques (retard mental, épilepsie), de même qu'il existe des recouvrements cliniques et nosographiques entre les désordres autistiques et d'autres troubles neurodéveloppementaux ou neuropsychiques, comme le déficit attentionnel avec ou sans hyperactivité, les syndromes dys- (dysphasie, dyslexie, dyspraxie...), ou encore les troubles obsessifs-compulsifs (voir par exemple Volkmar et Pauls, 2003 ; Tardif et Gepner, 2003).

Dès lors, de nouvelles améliorations significatives dans le traitement et la rééducation des personnes souffrant de désordres de la constellation autistique ne pourront émerger que d'une meilleure compréhension des causes et des mécanismes des désordres autistiques en général, et de leurs conséquences chez un individu en particulier. Car quelle que soit notre empathie, notre grille de lecture, notre compétence, nos ressources humaines, nos savoirs et savoir-faire, et même si tel syndrome autistique peut s'atténuer de jour en jour, de mois en mois, d'année en année, et que des progrès relationnels, communicatifs et cognitifs, minimes ou importants, sont en principe toujours possibles, l'énigme du noyau des désordres autistiques demeure.

Cette énigme constitue indiscutablement depuis 60 ans une source inépuisable de curiosité et de motivation pour les cliniciens et chercheurs à travers le monde, et nous a personnellement incité il y a 15 ans à nous intéresser de près à ces personnes, à démarrer des recherches expérimentales en neuropsychologie cognitive, et à poursuivre en parallèle nos réflexions théorico-cliniques sur le fonctionnement et les dysfonctionnements de l'esprit-cerveau humain (Gepner, 2003 ; Gepner \& Tardif, in press ; voir aussi dernière partie du présent article).

Ces enfants, adolescents et adultes que nous rencontrons quotidiennement en consultation, en 
centre de jour, SESSAD et écoles spécialisées, nous invitent moralement et épistémologiquement à mieux comprendre leurs troubles neuropsychopathologiques sousjacents, pour mieux les aider eux-mêmes, aider leurs parents désemparés et inquiets, et aider leurs soignants interrogatifs. Les aider à quoi ? A franchir, ou à tout le moins réduire ou éclairer la zone d'incertitude nous séparant de ces personnes différentes.

Car ce qui fascine, ou dérange, voire perturbe lorsqu'on rencontre un enfant autiste pour la première fois est le contraste entre sa présence physique et son apparente absence psychique, une présence à autre chose que soi. En observant plus attentivement ces enfants, on en vient généralement à se dire que le monde dans lequel ils vivent est manifestement différent du nôtre. En réfléchissant, on peut aussi en venir à se dire que probablement ils éprouvent, ressentent, mais aussi perçoivent et comprennent le monde environnant différemment de nous. Des êtres aux comportements aussi bizarres seraient-ils nos semblables ? Comme être humain communiquant, on ne se sent vraiment rassuré, et comme clinicien, on ne peut vraiment rassurer la famille d'un tel enfant, que lorsque la magie d'une rencontre avec lui s'opère : un regard, même furtif, un sourire, un jeu interactif, une imitation... Même avec des personnes ayant un syndrome d'Asperger, ou parfois avec le parent d'un enfant autiste, l'on perçoit quelques différences subtiles entre elles et nous, dans leur langage, leur langage des yeux, leur langage émotionnel, leur langage facial, l'empathie, ou le contact social. Mais jusqu'à quel point cette personne est-elle différente de nous ? Plusieurs voies, complémentaires l'une de l'autre, s'offrent à nous pour répondre à cette question: la première, sans doute la plus authentique et relevant d'une vérité subjective, qui produit un discours en première personne, nous vient des personnes autistes elles-mêmes, quand elles s'expriment directement sur ellesmêmes, ce qui est relativement rare et précieux ; une seconde voie procède du récit d'un parent ou de l'observation du clinicien, quelle que soit sa pratique ou son obédience, et produit un discours à la troisième personne, issu de l'intersubjectivité, intermédiaire entre subjectivité et objectivité ; une troisième voie enfin procède d'une logique expérimentale, et produit un discours plus général, plus global, moins singulier, à vocation d'objectivité scientifique ou statistique. Dans le présent article, nous aurons le souci de faire converger entre elles ces trois voies, et comme si elles représentatient 3 brins de connaissance, d'en constituer un écheveau qui sera notre fil conducteur.

\section{Comment est née l'idée de Malvoyance de l'É-Motion dans l'autisme ?}

Comme nous l'avons rappelé récemment (Gepner, 2005), plusieurs arguments nous avaient conduit à supposer que certains enfants autistes pourraient souffrir de désordres de la perception visuelle du mouvement. Nous avons appelé ce désordre de l'intégration du mouvement la Malvoyance du mouvement (Gepner, 2001, 2005) et plus récemment la Malvoyance de l'É-Motion (Gepner, Lainé \& Tardif, 2005; Gepner \& Tardif, in press), pour rendre compte du fait que les anomalies peuvent porter sur tout type de mouvement, qu'il s'agisse de mouvements physiques (les flux visuels environnementaux, les mouvements de l'environnement produits par nos propres déplacements, les mouvements des objets) ou de mouvements biologiques (ceux des êtres vivants animés), tels que les mouvements du visage, et notamment les mimiques faciales émotionnelles.

Ces différents arguments en faveur de troubles possibles de la perception visuelle du mouvement proviennent tout d'abord de l'observation clinique directe des enfants autistes et de l'observation des films familiaux, mais aussi de témoignages de personnes autistes dites de haut niveau, ou encore de la neuropsychologie adulte et de recherches expérimentales en neuropsychologie cognitive. Nous rappelons ces arguments, avant de présenter rapidement les travaux expérimentaux démontrant la réalité d'une malvoyance du mouvement chez certains enfants autistes, puis d'actualiser et compléter nos vues dans les paragraphes suivants. 


\section{Observation clinique}

En ce qui concerne l'observation clinique, Kanner avait noté chez presque tous les enfants de son observation princeps de 1943 plusieurs particularités comportementales qui ont un lien direct ou indirect avec le mouvement, qu'il s'agisse de la perception ou de la production de mouvement ou encore de couplage sensorimoteur. Sur le plan perceptif, certains enfants décrits dans son observation présentaient un évitement du regard ou du visage (signe quasipathognomonique de l'autisme, à propos duquel Temple Grandin (1995) écrit : "Il se pourrait que les problèmes de contact oculaire rencontrés par les autistes résultent en partie d'une incapacité à supporter le mouvement des yeux d'un interlocuteur. Un autiste a raconté qu'il lui était difficile de regarder les yeux de quelqu'un parce qu'ils n'étaient jamais immobiles ») ${ }^{1}$, une aversion pour les objets animés avec une attraction par le monde statique et une absorption dans les détails, les formes, les puzzles, ou a contrario une attraction pour certains objets en mouvement, tournoyants ou roulants; sur le plan moteur, certains enfants présentaient une maladresse motrice ou gestuelle, des déambulations sans but, des désordres sensorimoteurs comme un défaut de coordination oculomotrice, des défauts d'imitation, des mouvements inhabituels stéréotypés des mains, des bras ou du corps (flapping, balancements, tournoiements...). Ces symptômes, aversion et/ou attraction pour le mouvement, désordres sensorimoteurs et production de mouvements atypiques, plaident en faveur de particularités dans le traitement attentionnel, perceptif et intégratif du mouvement environnant physique et humain chez les premiers enfants décrits comme autistes dans la littérature.

Les cliniciens depuis 60 ans ont nécessairement été confrontés à de tels symptômes chez les enfants autistes qu'ils rencontrent : un courant de cliniciens psychanalytiques a interprété ces symptômes comme étant des manifestations d'angoisses archaïques, dont certaines avaient une connotation clairement perceptive, qu'il s'agisse par exemple des sensations de chutes sans fin et d'arrachement décrites par les patients suivis en cure intensive par Tustin (1972), ou du démantèlement perceptif de Meltzer et coll. (1980), ces angoisses suscitant secondairement des modalités de défenses propres aux enfants autistes (recours à un objet autistique selon Tustin, identification adhésive selon Meltzer). A lire les descriptions saisissantes de Grandin sur ses véritables attaques de panique liées à ses perceptions tactiles ${ }^{2}$ et en sachant qu'elle a inventé sa fameuse machine à serrer pour se soigner de ses désordres tactiles, on ne peut que reconnaître d'une part le lien puissant et indissoluble entre vécu sensoriel et vécu émotionnel, entre sensorialité et émotionnalité, et d'autre part la corrélation voire l'équivalence neuro-psychologique entre défense sensorielle et défense psychique, c'està-dire entre mécanismes préventifs à l'égard d'un excès de flux sensoriels (excès lié à l'intensité ou à la vitesse du flux), et mécanismes psychiques de contournement ou de scotomisation, de refoulement ou d'évitement des informations fournies par les sens via le cerveau.

\footnotetext{
${ }^{1}$ Le stimulus mouvement, le flux visuel, pourrait devenir (dans certaines conditions de fréquences temporelles et spatiales, donc de vitesse, puisque la vitesse s'exprime comme le rapport des fréquences temporelles sur les fréquences spatiales $\mathrm{V}=\mathrm{FT} / \mathrm{FS}$ ) un stimulus aversif, comme le sont certains stimuli sonores ou tactiles (voir plus loin).

${ }^{2}$ "Aussi loin que remontent mes souvenirs, j’ai toujours détesté que quelqu'un me prenne dans ses bras. Je voulais connaître le plaisir de l'étreinte, mais je reculais toujours. Un raz de marée de sensations déferlait et je réagissais comme un animal sauvage. Le contact physique déclenchait chez moi un réflexe de fuite; on aurait dit qu'il faisait disjoncter tout le circuit. Je devais fuir ce trop-plein d'émotions et je m'échappais, souvent en m'arrachant brusquement de l'étreinte... Depuis le début de la puberté, je vivais en permanence dans la peur et l'anxiété, avec des attaques de panique sévères survenant à des intervalles irréguliers...».
} 
D'un autre côté, il n'est pas étonnant que l'investigation de la signification neurophysiologique et neuropsychologique de ces symptômes soit encore assez réduite, car la grande majorité des travaux expérimentaux menés dans ce domaine depuis 40 ans dans la population autistique (y compris ceux effectués actuellement en neuroimagerie) ont été réalisés chez des individus autistes dits de haut niveau, ou présentant un syndrome d'Asperger, c'est-à-dire présentant essentiellement des anomalies socio-cognitives et dont les anomalies perceptives ou motrices sont relativement subtiles, et donc plus faciles à tester. Quelques cliniciens américains avaient pourtant précisément exploré les troubles perceptivomoteurs des enfants autistes et proposé des explications neurophysiologiques (inconstance perceptive, désordres d'intégration sensorimotrice, par défaut de modulation réciproque entre inputs sensoriels et outputs moteurs, Ornitz et Ritvo, 1968 ; Ornitz, 1974). Ils ont été suivis en France par l'équipe de Gilbert Lelord (1998), conduisant en particulier à l'observation clinique détaillée du bébé autiste ou à risque autistique.

\section{Clinique des films familiaux}

Depuis une vingtaine d'années en effet, quelques groupes, dont celui de Dominique Sauvage (Sauvage, 1988 ; Receveur et al., 2005) et de Teitelbaum et coll. (1998), ont utilisé l'analyse rétrospective des films familiaux pris au domicile par les parents d'un bébé dont ils apprendront par la suite qu'il présente un syndrome autistique, pour la recherche des signes les plus précoces d'autisme. Ces signes concernent les domaines perceptif, sensorimoteur, langagier et interactif chez des bébés de 0 à 24 mois.

Etant donné que le mouvement, physique et biologique, est -quasi- ubiquitaire et permanent depuis le début de la vie de tout individu, et qu'il est absolument crucial pour le développement de l'imagerie cérébrale du mouvement et de l'action, le développement posturomoteur, l'imitation, les interactions verbales et affectives, langagières et émotionnelles... (Gepner, 1997, 2001 pour une revue), il n'est pas difficile d'imaginer les conséquences développementales variées et envahissantes de désordres précoces de la perception du mouvement chez un bébé.

Parmi les principaux signes autistiques précoces relevés dans l'analyse des films familiaux, certains sont en rapport avec un possible désordre de la perception visuelle ou de l'intégration visuo-motrice du mouvement ${ }^{3}$. Ainsi, dès ses premières semaines de vie, le bébé à risque autistique peut présenter des anomalies du regard, du contact oculaire, et de la poursuite oculaire des objets ou personnes en mouvement. Dans le domaine moteur, le bébé autiste peut présenter des troubles d'ajustement postural (il est trop mou, ou trop raide). Jusqu'à 3 mois, les anomalies du regard persistent et le bébé autiste peut montrer plus nettement un défaut d'attention aux personnes, ainsi qu'un défaut de sourires et d'expressions faciales. Sur le plan de l'activité motrice, il est soit particulièrement sage, soit trop agité. Jusqu'à 6 mois, le défaut de contact visuel peut persister, avec un regard «vide», et parfois un strabisme. Ses expressions faciales sont pauvres. Il peut commencer à montrer un intérêt atypique pour ses mains, les détails, les formes statiques, avec en revanche un moindre intérêt pour les jouets en mouvement. Sur le plan moteur, il peut présenter une absence ou un retard d'attitude anticipatrice, une absence ou un défaut d'ajustement postural (poupée de chiffon ou raideur excessive), un retard d'acquisition de la station assise. Dans le second semestre, le bébé autiste

\footnotetext{
${ }^{3}$ Notons que ces signes, parfois très subtils, ne sont pas retrouvés de manière systématique dans le développement précoce d'enfants qui s'avéreront présenter ultérieurement un désordre du spectre autistique. En particulier, le développement du bébé peut s'avérer tout à fait normal dans les douze ou quinze premiers mois de sa vie, et régresser ensuite (cas du trouble désintégratif). Par ailleurs, certains de ces signes isolés peuvent s'atténuer, voire disparaître chez certains bébés, soit de manière probablement spontanée, soit au cours d'une intervention thérapeutique précoce.
} 
peut donner une impression de cécité ; son expression faciale est appauvrie, il n'imite pas, ou il imite peu et de façon déviante, les mimiques de son partenaire d'interaction. Il montre peu ou pas d'intérêt pour les personnes, il commence à s'isoler, se retirer de l'échange social et affectif. Parallèlement, il continue à développer des comportements inhabituels d'autostimulation sensorielle (visuelle ou proprioceptive), de type balancements, jeux de doigts ou de mains devant les yeux. Sur le plan moteur, il peut présenter un retard d'acquisition de la station debout. Dans la seconde année, le tableau autistique se complète par un défaut d'attention visuelle (regard périphérique, furtif) et d'attention conjointe, un défaut de contact avec les personnes (retrait, interactions pauvres), et par des intérêts particuliers pour les sources lumineuses, les reflets, les ombres, le vent dans les arbres...Sur le plan moteur, peuvent apparaître des défauts de coordination oculo-manuelle et d'exploration de l'espace, un retard d'acquisition de la marche, ainsi que des mouvements stéréotypés (battements des mains, balancements, tournoiements).

Ce survol rapide de la progression des signes autistiques précoces montre plusieurs choses d'importance pour notre propos : tout d'abord, les premiers signes qui apparaissent au cours de la vie d'un bébé à risque autistique concernent son développement visuel, avec probable dissociation entre sa vision du mouvement (atypique, aversive) et sa vision des formes statiques, normale voire surdéveloppée (avec attraction pour les détails, retrouvée plus tard chez des enfants et adultes, y compris ceux ayant un haut niveau de fonctionnement (Frith, 1989 ; Happé, 1999 ; Mottron, 2004). Ces premiers signes affectent aussi son développement visuo-posturo-moteur (défaut d'ajustement postural et d'anticipation motrice, dont on sait qu'il est fortement lié à un bon fonctionnement de la proprioception visuelle, c'est-à-dire intégrant les événements visuels dynamiques environnementaux dans le corps propre). Deuxièmement, parallèlement à un tableau déficitaire marqué par le retard de développement (que l'on pourrait nommer «signes négatifs», par analogie avec les signes négatifs de la schizophrénie), apparaissent des comportements visuels et visuo-moteurs atypiques (auto-stimulation sensorielle), évoquant une déviance de la trajectoire développementale, que l'on pourrait nommer «signes productifs » et qui, pour certains d'entre eux, pourraient avoir une fonction adaptative ou compensatoire. Troisièmement, il est possible de voir la progression des signes autistiques comme une succession de cascades «maldéveloppementales », où des comportements visuels altérés déforment secondairement les échanges visuels, posturomoteurs, mais aussi communicatifs et sociaux entre l'enfant autiste et son environnement humain et non-humain. Enfin, il manque dans cette description des signes autistiques précoces tous les éléments relatifs au domaine auditif, qui contribuent aussi aux désordres du développement du langage, et dont nous reparlerons plus loin.

\section{Témoignages d'autistes célèbres et moins célèbres}

Nous avons à plusieurs reprises rapporté les propos édifiants d'adultes autistes célèbres concernant les particularités de leur monde visuel, qui sont directement en rapport avec le mouvement visuel, la vitesse des changements du monde environnant, ou encore des stratégies de compensation de leurs désordres perceptifs.

Donna Williams, écrit dans Nobody nowhere (1992): "Le changement perpétuel qu'il fallait affronter partout ne me donnait jamais le temps de me préparer. C'est pourquoi j'éprouvais tant de plaisir à faire et refaire toujours les mêmes choses. (...) J'ai toujours aimé l'aphorisme "Arrêtez le monde, je veux descendre!" Est-ce pour avoir été absorbée dans les taches et les "étoiles" au moment précis où les autres enfants s'ouvrent au monde extérieur que je suis restée sur le bord de la route? Toujours est-il que la tension qu'exigeait la nécessité d'attraper les choses au vol pour se les assimiler fut le plus souvent trop forte pour moi. Il me fallut trouver un biais pour ralentir les choses afin de m'accorder le temps de négocier avec elles. (...) L'un des procédés qui me permettaient de ralentir le monde consistait soit à cligner des yeux, soit encore à éteindre et allumer alternativement la lumière 
rapidement. Si vous cligniez des yeux vraiment vite, vous pouviez voir les gens sautiller comme dans les vieux films; vous obteniez le même effet qu'avec un stroboscope, mais sans avoir besoin de manipuler quoique ce soit... ». Dans l'épilogue de son livre, Donna Williams ajoute un «Aperçu du langage particulier à mon propre monde » :

- Stéréotypes et comportements stéréotypés. Ils donnent un sentiment de continuité. Les rituels, les gestes stéréotypés donnent l'assurance que les choses peuvent rester les mêmes assez longtemps pour avoir leur place incontestée au sein d'une situation complexe et mouvante autour de soi.

- Cligner des yeux compulsivement. Cela permet de ralentir les choses et de les rendre plus fractionnées, donc moins effrayantes, comme dans un film qui passe au ralenti. Teindre et rallumer la lumière très vite a la même fonction.

- Eteindre et allumer la lumière. L'interrupteur est un signal impersonnel en relation avec le monde extérieur, comme les clochettes ou la musique. Cela donne le plaisir d'une sensation physique doublé d'un sentiment de sécurité, qu'il n'est pas possible d'atteindre par le contact physique direct. Cela rend les choses plus fixes, donc plus prévisibles et plus rassurantes. (Williams, ibid., p. 280-281).

Le point important du langage du corps de Donna Williams est sa difficulté à capter les changements incessants du monde environnant, l'insécurité et l'angoisse physique et psychique qui en découlent, et les stratégies comportementales qu'elle invente pour ralentir voire figer le monde afin de mieux s'y préparer, mieux le capter, et le rendre ainsi plus rassurant.

Temple Grandin écrit dans Thinking in pictures and other reports from my life with autism (1995) : "Il se pourrait que les problèmes de contact oculaire rencontrés par les autistes résultent en partie d'une incapacité à supporter le mouvement des yeux d'un interlocuteur. Un autiste a raconté qu'il lui était difficile de regarder les yeux de quelqu'un parce qu'ils n'étaient jamais immobiles ». Plus loin, elle écrit : "La formation d'images altérées explique peut-être la préférence de certains autistes pour la vision périphérique. Il est possible que ceux-ci reçoivent des informations plus fiables quand ils regardent du coin de l'oeil... Une personne autiste a raconté qu'elle voyait mieux en regardant de côté et qu'elle ne voyait pas les choses en les regardant directement... Les déficiences mineures du traitement des informations visuelles renforçaient mon attirance pour certains stimuli (j'adorais regarder les portes coulissantes des supermarchés ou des aéroports), qui auraient effrayé ou fait fuir un autre enfant atteint d'anomalies plus sévères ». Temple Grandin suggère elle aussi une stratégie de compensation de problèmes visuels possiblement liés à l'hypersensibilité au flux visuel rapide. La vision périphérique utilisée seule permettrait de minimiser l'excitation fovéale, de débarrasser les informations sur le mouvement des autres informations visuelles 'parasites', du bruit visuel. Comme porte-parole de ses «semblables », Temple Grandin souligne également les comportements visuels particuliers des personnes autistes comme un continuum partant de l'aversion à l'attraction pour le mouvement, selon le degré du désordre de traitement sensoriel (voir aussi la description de Temple Grandin par Sacks, 1996).

Lorsqu'on se tourne vers des personnes plus anonymes, l'on peut en apprendre davantage sur ce problème de malvoyance du mouvement. La mère d'une adolescente autiste de bas niveau m'a rapporté les propos suivants : "Ma fille a toujours eu un problème avec le mouvement. Elle a peur quand les gens s'approchent trop près d'elle, elle évite toutes les situations où les choses vont vite. Moi-même, lorsque je dois conduire une voiture, je n'excède jamais $70 \mathrm{~km} / \mathrm{h}$, car au-delà, je me sens mal, si bien que tout le monde me klaxonne. Ma mère n'a jamais conduit une voiture, car elle ressent le même inconfort, mais à un degré plus important, elle ne supporte pas de rouler à une vitesse supérieure à $40 \mathrm{~km} / \mathrm{h}$. Pour la même raison, elle ne regarde jamais la télévision, ne va jamais au cinéma, car tout va trop vite...". Ce type de témoignage d'une anomalie perceptive transgénérationnelle est particulièrement intéressant pour notre propos, et n'est sans doute pas unique. De nombreux parents rapportent chez leur enfant de sérieuses difficultés dans la gestion des événements dynamiques rapides (films, dessins animés, voitures dans la rue), des mouvements environnementaux (objets, balles ou ballons, déplacements des personnes), des séquences motrices rapides (course, vélo...), et certains d'entre eux évoquent des difficultés 
similaires dans leur propre enfance.

\section{Neuropsychologie adulte}

Nous avons également rapporté à plusieurs reprises cette extraordinaire étude de cas neuropsychologique adulte de Zihl, von Cramon et Mai (1983), dont l'intérêt est de nous montrer les conséquences d'une anomalie brutale de la perception visuelle du mouvement chez une adulte jusque là indemne de toute difficulté. Ces auteurs rapportent en effet le cas d'une jeune femme de 43 ans qui, à la suite d'une hémorragie cérébrale postérieure bilatérale, a présenté des troubles importants de la vision du mouvement dans les trois dimensions de l'espace. Elle percevait par exemple le flux du thé ou du café versé dans une tasse comme un solide gelé. De plus elle n'arrivait pas à s'arrêter de verser le liquide dans la tasse au bon moment car elle était incapable de voir le niveau du liquide monter. Cette patiente se plaignait aussi de ne pas arriver à suivre un dialogue parce qu'elle était incapable de capter les mouvements du visage et spécialement ceux des lèvres. S'il se trouvait plus de deux personnes dans une pièce, elle se sentait en insécurité, et quittait le plus souvent immédiatement la pièce : "les personnes se trouvaient soudain ici ou là, mais je ne les voyais pas se déplacer ». Cette patiente était confrontée au même problème dans les rues, mais à un degré bien plus marqué, si bien qu'elle s'était mise à les éviter. En effet, alors qu'elle identifiait bien les voitures, elle ne pouvait pas traverser la rue en raison de son incapacité à évaluer leur vitesse. "Quand je vois la voiture, elle semble loin. Puis quand je veux traverser la rue, soudain la voiture est tout près ».

Différents tests neuro-psychologiques effectués auprès de cette patiente ont montré chez elle une abolition complète de la vision du mouvement en profondeur. Pour des cibles visuelles se déplaçant en champ visuel central le long d'un axe vertical ou horizontal, la perception du mouvement était quelque peu préservée : la patiente pouvait distinguer différentes directions de mouvement, et juger la vitesse de la cible si celle-ci n'excédait pas $10 \mathrm{deg} / \mathrm{s}$. Par contre, dans le champ visuel périphérique, sa sensibilité au mouvement se limitait à pouvoir discriminer entre une cible mobile et une cible immobile. Ses mouvements de poursuite visuelle étaient corrects seulement pour des vitesses lentes (moins de $8 \mathrm{deg} / \mathrm{s}$ ). Ses mouvements visuellement guidés de poursuite d'un câble avec l'index de sa main droite étaient très altérés. La patiente commente sa pauvre performance en disant que sa difficulté se situe «entre son doigt et ses yeux. Je ne peux pas suivre mon doigt avec mes yeux si je bouge mon doigt trop vite ». Par contre, sa perception du mouvement en modalité tactile ou auditive était normale.

Cette étude neuropsychologique remarquable nous permet de mesurer l'ampleur des conséquences, sur les comportements perceptifs, visuo-moteurs, communicatifs et sociaux, d'un trouble pourtant électif de la perception du mouvement survenant brutalement chez une adulte jusque là indemne de toute difficulté. Cette patiente présentait en effet des altérations de la perception de la gestualité faciale (dont les mouvements labiaux), une maladresse visuomotrice, une appréhension des lieux animés (rues, pièces où il y a plus de deux personnes) et de grandes difficultés dans les interactions sociales, au point qu'elle les évitait le plus possible. Cette étude de cas peut dès lors nous aider à imaginer les différentes conséquences développementales d'un trouble qui affecterait la vision du mouvement chez un nourrisson ou un jeune enfant.

\section{Recherche en neuropsychologie cognitive : la question du traitement des visages}

Le dernier aspect qui nous a incité à rechercher des anomalies de la perception visuelle du mouvement est fondé sur nos recherches antérieures sur la reconnaissance des visages chez les enfants autistes. On sait que l'autisme est marqué par des troubles de la communication 
verbale et émotionnelle et de l'interaction sociale. Dans la mesure où le visage humain est la première et la plus puissante source d'informations médiatisant la communication verbale et émotionnelle et les interactions sociales, il n'est pas surprenant que le traitement des visages ait été souvent et régulièrement étudié dans la population autistique dans les trente dernières années (Dawson et al., 2005, pour une revue). En effet, un nombre croissant d'études en neuropsychologie cognitive a démontré que les personnes souffrant d'autisme traitent généralement les différents aspects des visages d'une manière différente de celle des personnes ordinaires ou présentant un retard mental. Des particularités ont été repérées dans le traitement de l'identité des visages (Langdell, 1978; Volkmar et al., 1989; Boucher \& Lewis, 1992; Davies et al.,1994; Klin et al.,1999; Deruelle et al.; 2004) et de l'expression faciale émotionnelle (Hobson, 1986a, 1986b; Hobson et al., 1988; Celani et al., 1999), dans la lecture sur les lèvres (de Gelder et al., 1991 ; Gepner et al., 1996) et la détection ou l'interprétation de la direction du regard (Gepner et al., 1996; Baron-Cohen et al., 1995). De récentes études en IRM fonctionnelle ont confirmé ces particularités chez les sujets avec autisme de haut niveau ou syndrome d'Asperger (Critchley et al., 2000; Schultz et al., 2000 ; Hadjikhani et al., 2004). Suivant cette ligne d'exploration neuropsychologique cognitive et développementale, deux études ont été conduites par notre groupe sur le traitement de 4 aspects des visages (l'identité faciale, l'émotion faciale, la direction du regard, le langage des lèvres), auprès d'enfants et adolescents autistes (Gepner et al., 1994 ; Gepner et al., 1996). Elles ont montré que les sujets autistes ont des difficultés plus ou moins importantes dans tous ces aspects du traitement des visages, et que ces difficultés sont liées soit à un défaut de traitement de la forme globale (la configuration du visages, résultat confirmé récemment par Deruelle et al., 2006), soit à un défaut de traitement de la dynamique faciale, impliquée dans les mimiques faciales émotionnelles et dans le mouvement des yeux et des lèvres.

L'ensemble de ces arguments nous a conduit à nous intéresser de près à cette question de la perception du mouvement dans l'autisme, qu'il s'agisse des mouvements physiques environnementaux ou des mouvements humains, car cette question semblait féconde d'un point de vue clinique, et prometteuse d'un point de vue expérimental et rééducatif.

\section{Recherches sur la perception et l'intégration du mouvement}

Nous résumerons dans ce paragraphe la littérature scientifique (notamment nos propres travaux) visant à attester directement des désordres de la perception visuelle et de l'intégration visuo-motrice du mouvement dans l'autisme. Nous le ferons brièvement puisque d'une part cette littérature est assez technique, et que d'autre part, nous l'avons déjà présentée de manière assez détaillée récemment (Gepner, 2005).

Comme Milne et al. (2005) le soulignent dans leur récente revue de la littérature sur ce sujet, notre groupe a été le premier il y a plus de 10 ans à se pencher sur la manière dont les enfants autistes perçoivent les mouvements et les intègrent dans leur corps propre.

Nous avons ainsi montré que des enfants autistes d'un niveau global pauvre ont une très faible réactivité posturale à la vision d'un mouvement ambiant, ce qui signifie chez eux une moindre dépendance posturale au mouvement visuel que chez des enfants ordinaires qui montrent quant à eux un véritable asservissement postural au mouvement visuel ambiant (Gepner et al., 1995). En effet, la vision est une source d'information importante pour le contrôle postural (Lee \& Aronson, 1974 ; Gibson, 1979), et de nombreuses études ont montré que de jeunes enfants réagissent posturalement au mouvement de leur environnement dès qu'ils tiennent debout sans aide (Butterworth \& Hicks, 1977) et même avant cela (Jouen, 1988).

Puis nous avons précisé que le même type d'enfants autistes ayant un niveau global pauvre et des anomalies posturo-motrices importantes montrent une certaine réactivité posturale au 
mouvement ambiant lorsque celui-ci est lent, mais que ces enfants ne réagissent plus posturalement lorsque le mouvement environnant est rapide, comme si, à partir d'un certain seuil de vitesse, les enfants autistes décrochaient par rapport au mouvement environnant, ce qu'on nomme le découplage visuo-postural (Gepner et Mestre, 2002a). Dans cette même étude, nous avons montré que des enfants avec syndrome d'Asperger (le plus léger des syndromes autistiques, sans retard verbal ni cognitif majeurs) montraient quant à eux une sorte d'hyper- ou de sur-couplage visuo-postural au mouvement environnant, par rapport aux enfants ordinaires, comme si leur appareil visuo-postural fonctionnait en sur-régime ou surcompensation ${ }^{4}$. Nous suggérions ainsi l'existence d'une corrélation entre l'intensité d'un syndrome autistique chez un enfant et le degré de ses difficultés visuo-posturales en réponse au mouvement ambiant. Comme l'écrit Grandin (1995), il existerait un parallèle entre le continuum des désordres du spectre autistique et le continuum des désordres perceptifs et sensori-moteurs.

Concernant la perception visuelle du mouvement en vision focale, nous avons montré que les enfants autistes avaient certaines capacités à comparer des vitesses de points sur ordinateur dans des gammes de vitesse lente et pour des trajets linéaires, et que plus les vitesses des points étaient élevées et/ou que leur trajet était complexe et imprévisible, plus leurs performances chutaient (Gepner, 1997).

Plus récemment, Bertone et al. (2003) ont montré que des enfants autistes de haut niveau présentent un déficit de la détection de la direction de mouvements transversaux, radiaires, ou de rotation de second ordre, résultat qui confirme une limitation de la perception visuelle du mouvement dans l'autisme.

$\mathrm{Au}$ moins quatre études ont montré que des enfants autistes de niveau variable ont une moindre réactivité visuo-oculomotrice en réponse à des mouvements de points sur un écran de grande dimension (Spencer et al, 2000 ; Milne et al, 2002 ; Pellicano et al., 2005), c'est-àdire une moindre capacité à extraire un mouvement global dans un stimulus bruité, et ce d'autant plus que les vitesses des points sont élevées (Mestre et al, 2002 ). En d'autres termes, les enfants autistes auraient un déficit de l'intégration spatio-temporelle de points singuliers dans un ensemble de points animés d'un mouvement cohérent. Ces études confirment avec un paradigme visuo-oculomoteur, les résultats obtenus avec le paradigme visuo-postural (Gepner et al., 1995 ; Gepner et Mestre, 2002a) qui suggèrent l'existence d'un déficit de la vision du mouvement chez les enfants autistes. Ces résultats sont compatibles avec la préférence souvent rapportée des personnes autistes pour un traitement local au détriment d'une intégration globale de l'information, appelée déficit de cohérence centrale (Frith, 1989; Happé, 1999). D'autres études confirment un déficit des mouvements de poursuite visuelle y compris chez des individus autistes de haut niveau (Takarae et al., 2004), bien que ce résultat ne soit pas retrouvé dans toutes les séries (Kemner et al., 2004).

Sur le plan des mouvements biologiques humains, nous avons démarré également une série d'études visant à évaluer l'influence du mouvement sur la reconnaissance des mimique faciales chez de jeunes enfants autistes. Nous voulions notamment savoir dans quelle mesure, en lui présentant lentement des expressions faciales (à contenu émotionnel ou non), on ne pourrait pas fournir à l'enfant autiste les moyens d'extraire une information qu'il semble avoir du mal à extraire à partir de mouvements plus rapides (cf. par exemple les descriptions autobiographiques de Williams, 1992). Les résultats d'une première étude (Gepner, Deruelle, Grynfeltt, 2001), montrent que les enfants autistes reconnaissent des mimiques faciales aussi bien que des enfants normaux plus jeunes, et qu'ils ont donc une aptitude à percevoir des

\footnotetext{
${ }^{4}$ Un des enfants ayant un syndrome d'Asperger était littéralement fasciné par ces sensations visuelles et demandait à l'expérimentateur de manipuler les images de tunnel visuel, de telle sorte que les impressions produites étaient celle d'un tunnel sans fin dans lequel on tombait inexorablement : était-ce une façon pour lui de retrouver une sensation connue de « chute sans fin »?
} 
mouvements faciaux présentés lentement sur vidéo, ou à tout le moins une aptitude à extraire une information faciale pertinente d'une séquence dynamique lente. Autrement dit, le mouvement lent semble offrir aux enfants autistes la possibilité d'extraire une information que, ni la présentation statique (les enfants autistes échouent généralement dans le même type de tâches présentées sur photographies), ni le mouvement trop rapide de la vie quotidienne, ne leur permettent d'extraire efficacement. Il n'est pas impossible que la présentation dynamique lente, par la modification discrète des relations spatiales entre les éléments du visage qu'elle suppose, sollicite moins le traitement visuel focal contraignant chez les enfants autistes (Frith, 1989 ; Happé, 1999), et sollicite davantage chez eux un traitement configural du pattern (c'està-dire un mode de traitement plus efficace pour reconnaître les visages d'un coup d'œil).

Dans une étude très récente, nous avons repris le paradigme de présentation dynamique des mimiques faciales émotionnelles et non-émotionnelles, mais en utilisant trois vitesses de présentation des mimiques (vitesse normale, vitesse lente, vitesse très lente), et en étudiant l'influence de la sonorisation de ces mimiques (Lainé, Tardif et Gepner, sous presse ; Tardif, Lainé, Rodriguez \& Gepner, in press). Nos résultats confirment d'une part que les enfants autistes reconnaissent mieux les mimiques faciales quand celles-ci sont ralenties, d'autre part que le ralentissement de ces mimiques et de leurs sons correspondants induisent chez eux davantage d'imitation faciale et vocale que chez les enfants ordinaires, et enfin que parmi l'ensemble des enfants autistes, ceux qui présentent le retard développemental le plus important et/ou le syndrome autistique le plus sévère, sont ceux qui bénéficient le plus du ralentissement des mimiques pour les identifier. Ainsi, premièrement, le degré de malvoyance du mouvement facial semble être corrélé au degré de sévérité du syndrome autistique, comme nous avions montré précédemment que semblait exister un corrélat entre le degré de réactivité posturale à la vision d'un mouvement physique environnemental et le degré de sévérité d'autisme (Gepner et Mestre, 2002a). Deuxièmement, le ralentissement d'événements faciaux et vocaux améliore les capacités de reconnaissance et induit l'imitation des expressions faciales chez des enfants autistes. Etant donnée la documentation de ces 30 dernières années sur les retards ou déviances imitatives chez les enfants autistes, (Smith \& Bryson, 1994 et Williams et al., 2004, pour des revues), et ce depuis le tout début de leur vie (Zwaigenbaum et al., 2005 ; Receveur et al., 2005), et tout particulièrement dans le domaine de l'imitation des expressions faciales (Hertzig et al., 1989; Loveland et al., 1994; Rogers et al., 2003), notre étude pourrait à l'avenir être intéressante dans une perspective rééducative. Nous sommes en effet actuellement en train de tester l'impact d'un logiciel de ralentissement simultané des mouvements faciaux et de la parole d'un interlocuteur sur les capacités de compréhension verbale et d'imitation d'enfants et adolescents autistes, retardés mentaux, et témoins ordinaires. Si les résultats de ces travaux étaient concluants, ils ouvriraient une piste majeure pour la rééducation des troubles de la communication verbale et émotionnelle chez les enfants autistes. Enfin, sur le plan neurobiologique, ce ralentissement des événements environnementaux pourrait faciliter la synchronisation neuronale dans le cerveau des personnes autistes, et constituer de facto un facteur synchronisant (Gepner et Tardif, in press ; Tardif et al., in press ; et voir plus loin).

Blake, Turner, Smoski et al. (2003) ont également montré que des enfants autistes présentent un déficit de la reconnaissance d'activités humaines comme courir, marcher, sauter..., présentées à partir de points lumineux animés, et ils en déduisent également un trouble de la vision des mouvements biologiques dans l'autisme.

Nous pouvons déduire différents éléments de compréhension neuropsychologique de l'autisme à partir de ces résultats expérimentaux.

D'une part, les défauts de perception visuelle et d'intégration visuo-motrice du mouvement physique environnemental pourraient rendre compte de manière privilégiée des anomalies 
précoces dans le développement de l'ajustement visuo-postural et de la posturo-motricité 5 . D'autre part, les défauts perceptif visuel et d'intégration visuo-motrice des mouvements biologiques humains, corporels, gestuels, faciaux, pourraient quant à eux rendre davantage compte de leurs problèmes d'anticipation motrice, de lecture des visages, d'imitation faciale et corporelle, de communication verbale et émotionnelle et d'interaction socio-affective, qui apparaissent donc secondaires aux désordres perceptifs (Gepner, 2004 ; voir Figure 1) ${ }^{6}$. La continuité étant co-substantielle au mouvement, ces défauts perceptifs et intégratifs du mouvement pourraient également expliquer les difficultés d'accès à la conscience de la continuité et de l'unité corporelle chez certains enfants autistes (voir dernière partie de l'article). Le facteur vitesse du mouvement semble critique pour les enfants autistes : pour certains d'entre eux, hypersensibles au mouvement, plus la vitesse du mouvement augmente, plus le mouvement devient aversif ; pour d'autres, plus le mouvement est rapide, moins il est perçu, ce que nous avons résumé par le concept de déficit d'intégration du mouvement visuel rapide (Gepner et Mestre, 2002b); pour d'autres enfin qui, initialement, sont parfois mais pas toujours d'un meilleur niveau perceptivo-cognitif, il semble y avoir possibilité d'adaptation, d'habituation, voire de recherche de mouvements rapides (Gepner, 2004).

Pour rendre compte de ces anomalies perceptives et intégratives du mouvement physique et humain (notamment des mimiques faciales émotionnelles), nous avons proposé le concept de Malvoyance $d u$ mouvement, et plus récemment de Malvoyance de l'É-Motion dans les désordres de la constellation autistique.

${ }^{5}$ D'un point de vue neurophysiologique, ces anomalies perceptives visuelles et visuo-motrices impliquent au minimum i) la voie visuelle magnocellulaire, qui véhicule les informations sur le mouvement (analyse du flux visuel), la profondeur, les fréquences spatiales basses et la forme globale, depuis la rétine jusqu'au corps genouillé latéral puis au cortex visuel primaire (Livingstone \& Hubel, 1988 ), ii) la voie visuelle dorsale, qui prolonge la voie visuelle magno-cellulaire en distribuant ensuite ces informations visuelles depuis V1 jusqu'aux différentes structures corticales et sous-corticales avec lesquelles elle établit de nombreuses connections, principalement les cortex temporal, pariétal, préfrontal et frontal, le cervelet, les structures thalamiques mésencéphaliques et pontiques (BoisacqSchepens et Crommelinck, 1994). Parmi ces connections, les voies visuo-cérébelleuses seraient particulièrement impliquées, dans la mesure où le cervelet traite spécifiquement la vitesse des informations visuelles dynamiques (Johnson et Ebner, 2000), et où le vermis cérébelleux dorsal (lobules VI et VII) est fréquemment retrouvé hypo- ou hyperplasique chez les sujets autistes (Courchesne et al., 1994), avec possible perte du nombre de cellules de Purkinje (Courchesne, 1997), et enfin iii) les voies cérébello-prémotrices et cérebello-motrices (cérébello-préfrontales et frontales, qui sont responsables, avec les ganglions de la base, du contrôle et du réglage précis en temps réel des outputs moteurs (Doya, 2000), ainsi que les projections du cervelet sur les cortex pariétal et temporal qui participent à l'apprentissage moteur et cognitif (Middleton et Strick, 2000).

${ }^{6}$ Aux voies neurophysiologiques précédentes s'ajoutent celles mettant en jeu le «cerveau social », qui implique notamment l'amygdale spécialisée dans le décodage des signaux socio-émotionnels (Adolphs et al., 1994), le gyrus fusiforme qui traite les visages (Haxby et al., 2001), le sillon temporal supérieur qui traite les mouvements biologiques (Allison et al., 2000) ainsi que la voix humaine (Belin et al., 2000), ou encore les neurones miroirs qui sont situés à l'interface de la perception et de l'action (Rizzolatti et al., 1996), soit autant de territoires affectés dans l'autisme (voir respectivement BaronCohen et al., 2000 ; Critchley et al., 2000 et Schultz et al., 2000 ; Castelli et al., 2002 et Gervais et al., 2004 ; Oberman et al., 2005)... 


\section{Désordres du traitement temporospatial des événements multisensoriels dans l'autisme}

Dans ce paragraphe, nous présentons de nouveaux arguments cliniques, autobiographiques et expérimentaux qui mettent en évidence que la Malvoyance du mouvement rapide, ou la Malvoyance de l'É-motion, est un exemple particulier, mais pas le seul, d'anomalie du traitement temporospatial des événements ou flux sensoriels dans l'autisme.

\section{Arguments cliniques}

L'analyse sémiologique des comportements autistiques révèle que, en dehors du domaine visuel, d'autres domaines sensoriels peuvent être affectés chez les enfants autistes, voire chez un même enfant. En particulier, les sphères auditive et tactilo-kinesthésique sont très souvent altérées. Ainsi, on note fréquemment chez les personnes autistes, et ce parfois dès les premiers mois de vie, une indifférence au monde sonore, une insensibilité à la voix, voire une impression de surdité ou au contraire une hypersensibilité aux sons (avec tendance à se boucher les oreilles) ou des réactions paradoxales à certains sons; le langage est parfois absent, pauvre, apparemment non communicatif ou perturbé, et la compréhension verbale semble parfois profondément altérée.

Sur le plan tactilo-kinesthésique, on note fréquemment dans les antécédents d'une personne autiste une absence ou un défaut d'ajustement postural lors des têtées ou du portage ("poupée de chiffon' ou raideur excessive), une aversion pour le contact avec certains stimuli, notamment l'eau ou certains vêtements, une tendance à se dévêtir, une recherche des endroits contenants et générateurs d'une pression plus ou moins importante.

\section{Arguments autobiographiques}

Temple Grandin (1995) décrit dans le chapitre «La machine à serrer » ses propres troubles sensoriels ou ceux qui lui ont été rapportés par d'autres personnes autistes. Sur le plan tactile, elle écrit :

"Aussi loin que remontent mes souvenirs, j'ai toujours détesté que quelqu'un me prenne dans ses bras. Je voulais connaître le plaisir de l'étreinte, mais je reculais toujours. Un raz de marée de sensations déferlait et je réagissais comme un animal sauvage. Le contact physique déclenchait chez moi un réflexe de fuite; on aurait dit qu'il faisait disjoncter tout le circuit... Si on nous touche sans que nous nous y attendions, nous nous replions sur nous-mêmes, parce que notre système nerveux n'a pas eu le temps de traiter l'information. Une femme autiste m'a confié qu'elle aimait beaucoup le contact physique, mais qu'il fallait que ce soit elle qui l'initie pour avoir le temps d'en intégrer la perception... Quand j'avais six ans, je m'enveloppais de couvertures et je me glissais sous les coussins du canapé parce que la pression me détendait... Depuis le début de ma puberté, je vivais en permanence dans la peur et l'anxiété, avec des attaques de panique sévères... Je passais mon temps à imaginer les moyens d'éviter les situations susceptibles de déclencher ces attaques... Quand j'étais jeune, j'avais besoin d'une pression très forte, presque douloureuse...L'intensité de la pression recherchée par une personne dépend peut-être de l'état d'excitation dans lequel se trouve son système nerveux...Certains autistes ont beaucoup de mal à sentir les limites de leur corps...Donna Williams a décrit une perception morcelée de son corps..Elle se tâtait et se frappait parfois pour trouver les limites de son corps. Quand elle éprouvait une trop forte quantité de sensations douloureuses, elle se mordait, sans s'apercevoir qu'elle venait de mordre son corps... ».

Sur le plan des troubles auditifs, elle écrit :

"Quand j'étais petite, le bruit était une source permanente de problèmes. C'était comme si la roulette $d u$ dentiste avait touché un de mes nerfs. Cela provoquait une réelle souffrance. J'avais une peur bleue des ballons qui éclatent...Quand j'étais à l'université, le sèche-cheveux de ma camarade de chambre me semblait faire le même bruit qu'un avion à réaction au décollage... Les enfants autistes ne supportent vraiment pas les bruits aigus et stridents, comme ceux d'une perceuse électrique, d'un 
robot de cuisine, d'une scie ou d'un aspirateur... La nature des sons qui dérangent varie d'une personne à l'autre. Un bruit qui me paraissait douloureux peut être agréable pour un autre enfant... Jane Taylor Mc Donnell raconte qu'on soupçonnait son fils de ne pas entendre certaines tonalités et certaines fréquences... Lorsque je me trouve dans une pièce bruyante, je n'arrive pas à comprendre ce qui se dit, car je ne filtre pas le bruit de fond. Quand j'étais petite, les fêtes de famille bruyantes m'affolaient; je n'arrivais plus à me contrôler et je piquais des crises de colère... Mes troubles auditifs sont légers comparés à ceux des autistes plus sévèrement atteints. Certains ont perdu la totalité ou la quasi-totalité de leur aptitude à comprendre le langage. D'autres ont l'oüe si fine que les bruits quotidiens leur sont insupportables. Un autiste a ainsi raconté que le bruit de la pluie ressemblait à une série de coups de feu... Leur monde se compose d'une masse de bruits confuse... Les enfants autistes atteints d'écholalie répètent ce qui a été dit afin de faciliter leur propre compréhension... Therese Joliffe (une adulte autiste) a expliqué qu'elle manquait presque toujours les premiers mots d'une conversation parce que comprendre que quelqu'un était en train de parler lui prenait du temps...».

Ces quelques éléments permettent d'éclairer de l'intérieur le monde sensoriel des personnes autistes, le long d'un continuum de troubles sensoriels, marqués par l'hypo- et/ou l'hypersensibilité à certaines intensités ou fréquences de stimulations acoustiques ou tactiles. Ces désordres sensoriels sont trop souvent négligés dans la compréhension psychopathologique des enfants autistes, alors qu'ils sont vraisemblablement une des voies privilégiées d'accès à leur être profond, et qu'une approche unitaire de ces désordres sensoriels pourrait être fondée sur une anomalie du traitement temporospatial des flux sensoriels.

\section{Arguments expérimentaux}

Afin d'explorer l'hypothèse du désordre du traitement temporospatial des événements multisensoriels dans l'autisme, nous avons testé, au sein d'un même groupe de 22 enfants et adolescents autistes, la capacité à extraire en temps réel une information pertinente au sein d'un stimulus bruité, dans trois types de tâches expérimentales : a) la réactivité oculo-motrice à un mouvement cohérent de points lumineux, à travers la mesure du nystagmus optocinétique ; b) la perception et la segmentation du flux verbal à travers la catégorisation de phonèmes simples et complexes ; c) la réactivité proprioceptive et l'anticipation motrice dans une tâche de délestage bimanuel, à travers la mesure d'indices électromyographique et cinématique. Les résultats de ce programme de recherche sont les suivants (Gepner et Massion (dirigé par), 2002) :

Comme mentionné plus haut (voir Mestre et al., 2002), le groupe des enfants avec autisme a montré une très faible réactivité oculomotrice au mouvement cohérent et des seuils plus élevés de cohérence du mouvement (c'es-à-dire la nécessité d'un pourcentage plus élevé de points allant dans une direction donnée par rapport à l'ensemble des points) par rapport au groupe des enfants ordinaires du même âge, comme cela avait été montré précédemment (Spencer et al., 2000 ; Milne et al., 2002). Cette anomalie, qui suppose un défaut d'analyse temporelle rapide de stimuli visuels en mouvement inclus dans du bruit, est un argument fort en faveur d'une dégradation de l'intégration temporospatiale en modalité visuelle chez les enfants autistes.

Deuxièmement, le même groupe d'enfants autistes a montré un déficit de la catégorisation de certains phonèmes. En effet, comparés à des enfants témoins ordinaires qui catégorisent un phonème ambigu comme MNA (qui est constitué d'une superposition algorithmique de phonèmes MA et NA) en une réponse MA ou NA au hasard (avec environ 50\% de MA et $50 \%$ de NA), les enfants autistes sur-catégorisent MNA en une réponse NA. Cette surcatégorisation anormale apparaît chez les enfants autistes quand les phonèmes sont présentés en vitesse normale, mais elle disparaît quand les phonèmes sont présentés en vitesse ralentie par deux. Ce défaut de catégorisation pourrait donc en partie être dû à une difficulté à 
traiter le flux verbal rapide, et donc à un défaut d'intégration temporelle dans la modalité auditive (Tardif et al., 2002). Un désordre du traitement temporel analogue a été trouvé il y a 30 ans chez des enfants ayant des troubles de développement du langage (dysphasie, dyslexie) par Tallal (1976); il a été relié à un défaut de fonctionnement du système visuel et/ou auditif magnocellulaire (Talcott et al., 2000 ; Stein, 2001), et a également été amélioré par un ralentissement de la parole (Tallal et al., 1996; Merzenich et al., 1996; Habib et al., 1999).

Enfin, il est apparu qu'un sous-groupe de ces mêmes enfants autistes présente un défaut d'anticipation motrice dans la tâche de délestage bi-manuel (Schmitz et al., 2002), un résultat qui confirme une étude précédente (Schmitz et al., 2003). Cette tâche requiert le traitement temporel rapide des événements proprioceptifs, la constitution d'un modèle interne (c'est-àdire d'une représentation interne du poids à délester), et l'ajustement temporel précis des événements musculaires visant à compenser le délestage. Comparés aux enfants témoins ordinaires qui utilisent un mode de contrôle anticipatif pour stabiliser leur avant-bras lors du délestage de l'objet placé sur le poignet, les enfants autistes utilisent un mode de contrôle rétroactif (en feedback), ce qui se fait au prix d'un ralentissement du mouvement. En d'autres termes, les enfants autistes réagissent au lieu d'anticiper. Ce défaut d'anticipation et d'ajustement temporel précis du contrôle du mouvement pourrait en partie résulter d'une altération du traitement temporospatial des événements proprioceptifs initiaux, et ainsi d'un défaut d'intégration temporospatiale dans la modalité proprioceptive.

Globalement, ces résultats suggèrent fortement que les enfants autistes ont un défaut d'intégration temporospatiale des flux sensoriels nécessaire pour détecter et intégrer le mouvement visuel, coder et découper le langage, et programmer des ajustements posturaux. Pour résumer, le monde semble aller trop vite, ou changer trop vite pour au moins certaines personnes autistes. Cette approche unitaire des désordres autistiques pourrait rendre compte de l'évitement sensoriel des personnes autistes (quand le flux sensoriel est aversif), et secondairement de leur évitement social, mais aussi du découplage perception-action et de leur désaccordage sensorimoteur, de leur désordres de compréhension verbale et émotionnelle, et in fine de leurs anomalies de compréhension du monde physique et humain qui les entoure et de leur désaccordage social et affectif (voir Figure 1).

\section{Synthèse, hypothèses neurobiologiques et parentés physiopathogéniques}

Notre approche synthétique des désordres de la constellation autistique est donc celle d'un trouble du traitement temporospatial des flux multisensoriels, dont la Malvoyance de l'ÉMotion est un exemple particulièrement saillant. Selon cette approche théorico-clinique, les personnes autistes souffriraient de troubles plus ou moins envahissants du traitement temporospatial des événements multisensoriels, c'est-à-dire d'anomalies dans la perception en ligne des événements visuels, auditifs et tactilo-kinesthésiques en provenance du monde environnemental, ainsi que dans la synchronisation de ces événements sensoriels, dans le couplage sensorimoteur et la production en temps réel d'événements moteurs et de communication verbale et émotionnelle, et ce dès les débuts de leur vie post-natale (Gepner, 2005; Gepner et al., 2005; voir Figure 1).

\section{Placer ici Figure 1}

Quelles pourraient être les bases neurobiologiques de tels désordres neuropsychologiques ? A un niveau neurofonctionnel, nous avons précédemment proposé (Gepner et Massion (dirigé par), 2002) que cette anomalie du traitement temporospatial pourrait être liée à un défaut de codage temporel des événements sensoriels et moteurs et de couplage sensorimoteur dans lequel le cervelet, véritable horloge du cerveau (Ito, 1984; Massion, 1993, 1997; Doya, 2000; 
Johnson \& Ebner, 2000; Middleton \& Strick, 2000 ), jouerait un rôle crucial. La notion de codage désigne le message contenu dans la décharge des neurones. La notion de codage temporel suppose que les caractéristiques temporelles ou fréquentielles de la décharge neuronale contiennent des signaux pertinents pour le fonctionnement du système nerveux. Le terme de codage temporel peut présenter plusieurs sens. Au niveau des ensembles neuronaux, ce terme donne à la synchronisation dans le temps des décharges neuronales de plusieurs sous-systèmes neuro-fonctionnels, la propriété de constituer de facto une cohérence du monde par simultanéité (Berthoz, 1997).

Cette explication est compatible avec l'hypothèse du déficit de liaison temporelle dans l'autisme (Brock et al., 2002), selon laquelle les individus autistiques souffriraient d'un déficit de la synchronisation des activités électriques à haute fréquence (activité gamma) ${ }^{7}$ entre des réseaux de neurones distants. Plus précisément, et selon l'hypothèse de Welsh et al. (2005), des perturbations dans la structure anatomique de l'olive inférieure, et donc dans la liaison olivo-cérebelleuse, telles que découvertes dans l'autisme (Bailey et al., 1998; Kemper \& Bauman, 1993), empêcheraient les neurones de l'olive inférieure de se synchroniser électriquement et de générer une sortie rythmique cohérente, ce qui altérerait la capacité des personnes autistes à traiter des informations rapides, et provoquerait chez elles un ralentissement de leur traitement cognitif global. Un résultat récent, montrant que des adultes avec un syndrome d'Asperger ont un retard d'activation corticale entre les zones occipitales, le sillon temporal supérieur, le lobe pariétal inférieur et le lobe frontal inférieur quand ils imitent des photos de formes de lèvres (Nishitani et al., 2004), va bien dans le sens de cette hypothèse. En suivant Welsh et al. (2005), et en s'appuyant sur de nombreuses données de la littérature sur la synchronisation neuronale dans le cerveau de l'animal et de l'homme (Varela et al., 2001, pour une revue), une information sensorielle rapide (un flux sensoriel rapide) parviendrait trop rapidement au cerveau des personnes autistes pour être traité en temps réel, ce qui altérerait la décharge simultanée (la synchronisation) de neurones d'une même assemblée, et entraînerait une désorganisation, une désynchronisation et un ralentissement du traitement de l'information. Une synchronisation neuronale anormale dans un réseau neuronal au sein d'une modalité sensorielle entraînerait secondairement un désordre dans le couplage temporel entre des assemblées neuronales de différentes modalités sensorielles.

Dans une étude récente, cependant, et contrairement à leur propre prédiction, Brown et al. (2005) ont montré que des sujets autistes présentent une activité gamma de synchronisation neuronale accrue lorsqu'ils doivent identifier la présence ou l'absence de formes illusoires de Kanisza.

Nous proposons donc que, selon le type de stimuli auquel elles sont exposées, les personnes autistes souffrent soit d'un défaut de synchronisation neuronale (ou désynchronisation neuronale) comme par exemple pour des stimuli dynamiques rapides ou globaux incluant le contexte, soit d'un excès de synchronisation neuronale (ou hyper-synchronisation neuronale) pour des stimuli statiques ou locaux, des détails, des singularités. Dans la mesure où la synchronisation neuronale est un mécanisme neurobiologique crucial dans les processus attentionnels, perceptifs et cognitifs (voir note 7), ces désordres de la synchronisation seraient

\footnotetext{
7 L'activité gamma correspond à des rythmes électrophysiologiques de fréquence élevée (30-100 Hz). L'amplitude de l'oscillation gamma est corrélée à la taille de l'assemblée de neurones synchronisés, et au degré de précision de la synchronisation. L'activité gamma est un marqueur de la synchronisation des décharges neuronales de différents réseaux neuronaux locaux spécialisés, de l'intégration temporelle des informations multi-sensorielles. Elle est notamment un marqueur neurophysiologique de l'attention consciente, de la mémoire de travail, de l'appariement des concepts, de la décision lexicale, de la perception consciente d'une forme globale (Rodriguez et al., 1999 ; Varela et al., 2001 pour une revue)...
} 
secondairement responsables des anomalies attentionnelles, perceptives et/ou cognitives, par défaut ou par excès, des personnes autistes.

Par ailleurs, l'hypersynchronisation pourrait apparaitre au cours du développement comme un mécanisme neuropsychologique destiné à compenser les effets d'une désynchronisation préalable, mais qui en quelque sorte dépasserait sa cible. En particulier, un phénomène d'hypersynchronisation au sein d'un réseau neuronal local puis entre des réseaux neuronaux distants, qui constitue le mécanisme d'épilepsie partielle et de généralisation (selon le modèle neuromimétique de Percha et al., 2005), serait tout à fait compatible avec la très fréquente association entre l'autisme et l'épilepsie clinique ou infraclinique (Hughes \& Melyn, 2005).

Nous proposons ainsi l'idée de dys-synchronie multisystème (au sein et/ou entre des réseaux neuronaux et des voies neurofonctionnelles plus ou moins distants) comme mécanisme fondamental des désordres de la constellation autistique. Cette dyssynchronie affecterait initialement un ou plusieurs systèmes-clés tels que le système visuel magnocellulaire (Gepner et Mestre, 2002b; Deruelle et al., 2004), le cervelet (Courchesne, 1997), la voie dorsale (Villalobos et al., 2005), le sillon temporal supérieur (Gervais et al., 2004), les neurones miroirs (Oberman et al., 2005...), puis, par effet tache d'huile ou boule de neige, vers l'amont et vers l'aval, elle s'étendrait éventuellement à d'autres réseaux neuronaux et voies neurofonctionnelles.

De plus, de nombreuse données issues de la physiologie humaine et animale (Varela et al., 2001, pour une revue), de la psychophysiopathologie (Babiloni et al., 2004 pour l'épilepsie; Symond et al., 2005 pour la schizophrénie; Just et al., 2004 pour l'autisme) et des modèles neuromimétiques (Borgers \& Kopell, 2003; Breakspear, 2004) ont montré l'étroite corrélation, l'interdépendance fonctionnelle voire l'équivalence entre synchronisation neuronale, rythmicité cérébrale et connectivité fonctionnelle (qui représente la corrélation spatiale par co-activation entre aires cérébrales). Autrement dit, la synchronisation est le mécanisme de la connectivité temporelle.

La contrepartie spatiale de cette dyssynchronie multisystème est donc une dysconnectivité multisystème (hypo- et/ou hyper-connectivité) au sein d'un réseau et/ou entre des réseaux neurofonctionnels multiples. Les recherches neuroscientifiques sur l'autisme de ces 30 dernières années ont révélé que de nombreuses zones cérébrales étaient touchées d'une manière ou d'une autre chez les personnes autistes. Mais la découverte d'un défaut de connectivité entre différentes aires cérébrales est plus récente (Horwitz et al., 1988). L'existence d'une sous- ou sur-connectivité entre réseaux neuronaux, déjà prévue par l'approche épigénétique et auto-organisationnelle des troubles autistiques (Gepner et Soulayrol, 1994), a été modélisée (Cohen, 1994). Actuellement, un nombre croissant d'études en IRM fonctionnelle (Courchesne \& Pierce, 2005; Belmonte et al., 2004), portant notamment sur le traitement des visages (Hadjikhani et al., 2004; Pierce et al., 2004) ou la compréhension de phrases (Just et al., 2004), tendent à montrer que c'est davantage la modulation d'activité entre régions cérébrales ou la connectivité entre ces régions, qui est altérée dans les désordres de la constellation autistique (Wickelgren, 2005 pour une revue). L'existence d'une dysconnectivité cérébrale dans l'autisme au cours de tâches de traitement de mimiques faciales émotionnelles vient d'être clairement confirmée par notre groupe (Wicker et al., submitted).

Cette dysconnectivité fonctionnelle serait au moins partiellement due à des mutations de gènes situés sur le chromosome $X$ et codant pour les neuroligines 3 et 4 , qui sont normalement impliquées dans l'adhésion cellulaire et la synaptogenèse (Jamain et al., 2003). En bref, nous pensons que les difficultés des personnes autistes à percevoir les événements ou flux sensoriels en ligne (c'est-à-dire au moment où ils leur parviennent), à intégrer ces flux dans leur corps propre, à coupler en temps réel perception et action, et à s'accorder cognitivement et émotionnellement à autrui dans les échanges communicatifs et sociaux, 
pourraient être une traduction comportementale et neuropsychologique de cette dyssynchronie et dysconnectivité multisystème, que celles-ci soient d'ordre structural et/ou fonctionnel.

Pour finir, et afin de rendre compte de la parenté entre certains signes cliniques présents dans les désordres autistiques d'un côté, et les syndromes dys- (c'est-à-dire dysphasie, dyslexie, dyspraxie...Habib, en préparation), les troubles attentionnels avec ou sans hyperactivité, et les syndromes épileptiques, d'un autre côté, nous suggérons que ces divers troubles neurodéveloppementaux partagent sans doute au moins un mécanisme physiopathogénique commun. Nous proposons que ce mécanisme physiopathogénique commun, permettant d'expliquer cette tendance à la comorbidité voire à certains recouvrements nosographiques entre ces différents syndromes, porte sur la synchronisation/connectivité neuronale. En effet, comme nous l'avons souligné plus haut, l'existence de troubles du traitement temporel a été démontrée il y a 30 ans chez certains patients atteints de troubles de développement du langage (dysphasie, dyslexie : Tallal, 1976), des désordres de la connectivité cérébrale ont été démontrés chez des sujets atteints de troubles attentionnels (Roessner et al., 2004), et les syndromes épileptiques sont typiquement marqués par une tendance à l'excès de synchronisation neuronale. Mais des troubles de la synchronisation sont également à l'œuvre dans la schizophrénie (Spencer et al., 2004) et la dépression (Tekell et al., 2005).

Que conclure de ceci ? Nous pensons qu'aussi différents soient-ils sur le plan clinique, les différents troubles neurodéveloppementaux, ou les différentes maladies neuro-psychiques, pourraient comporter dans leurs soubassements neurobiologiques fondamentaux, une altération portant sur la synchronisation/connectivité neuronale. Cette altération, en fonction de ses origines, de sa nature, de son intensité, de ses localisations, de son extension temporospatiale, se manifesterait par des expressions phénotypiques variées, plus ou moins regroupées dans différentes constellations cliniques.

Cette vision relativise, c'est-à-dire met en relation, l'autisme par rapport aux autres désordres neuropsychiques, ce qui ne doit pas empêcher (au contraire) de continuer de spécifier les différences entre les divers désordres de la constellation autistique, et entre ceux-ci et les autres désordres neurodéveloppementaux et neuropsychiques.

\section{Perspectives psychologiques, métapsychologiques et philosophiques}

Dans ce dernier paragraphe, nous essayons d'explorer les possibles conséquences de notre théorie des désordres de l'intégration temporospatiale des événements multisensoriels dans l'autisme sur le développement et le fonctionnement de la pensée, et nous donnons nos vues personnelles sur ce qu'on pourrait appeler la «personnalité autistique ».

Dans ce paragraphe également, nous proposons une vue peu orthodoxe de l'autisme en tant que modèle de dissociation psychisme-cerveau, et suggérons un continuum entre pensée, langage et action en termes de degrés d'énergie et de matérialité.

\section{(1) Continuum autistique et continuité de la pensée}

Quand elle décrit sa manière de pensée, Temple Grandin (1995) écrit : "Je pense en images. Pour moi, les mots sont comme une seconde langue. Je traduis tous les mots, dits ou écrits, en films colorisés et sonorisés; ils défilent dans ma tête comme des cassettes vidéo...Quand je lis, je traduis les mots en films en couleurs, ou bien je stocke simplement la photo de la page imprimée pour la lire plus tard. Quand je cherche dans ma tête, je vois la photocopie de la page. Il est probable que Raymond, l'autiste de haut niveau dépeint dans le film Rain Man, utilise une méthode semblable pour mémoriser les annuaires téléphoniques, les cartes routières et les autres informations. Il photocopie simplement chaque page du livre dans sa mémoire. Quand il a envie de retrouver un numéro, il balaie les pages de l'annuaire dans sa tête. Pour retrouver des informations dans ma mémoire, je dois repasser la 
cassette vidéo. Il est parfois difficile de retrouver certaines données parce qu'il faut que j'essaie différentes cassette jusqu'à ce que je trouve la bonne. Et cela prend du temps... Un autre fait atteste de l'utilisation de la pensée visuelle comme mode principal du traitement d'informations. C'est l'aptitude remarquable qu'ont beaucoup d'autistes à faire des puzzles, à s'orienter dans une ville ou à mémoriser un très grand nombre de données en un coup d'œil. Le cours de mes propres pensées ressemble à celui décrit par A.R. Luria dans Une mémoire prodigieuse. Ce livre raconte le cas d'un journaliste-reporter qui accomplit des prouesses avec sa mémoire. Tout comme moi, l'homme conserve une image visuelle de tout ce qu'il a lu ou entendu... Je continue de ne penser qu'en images, même si la pensée est de moins en moins visuelle à mesure qu'on s'éloigne de l'autisme de Kanner sur le continuum... J'ignorais que mon mode de pensée visuel était à l'origine de mes difficultés d'interaction avec autrui ».

Lorsqu'on lit ces descriptions une fois encore extraordinaires de Temple Grandin, et qu'on les met en rapport avec son monde sensoriel (voir paragraphes précédents), on comprend mieux le lien entre les particularités de son traitement sensoriel du monde environnant et son mode de pensée.

Nous avons déjà souligné dans les paragraphes précédents, à côté de Grandin, le parallèle (et probablement la corrélation) existant entre intensité du syndrome autistique et intensité des désordres sensoriels, avec à un bout du continuum les syndromes autistiques sévères marqués par de nombreux désordres sensoriels profonds et envahissants, et à mesure que l'on s'éloigne de ces tableaux cliniques, une diminution de l'intensité des désordres sensoriels, et à l'autre extrêmité du continuum, le syndrome d'Asperger marqué par des difficultés principalement socio-cognitives. Selon l'idée communément admise que le mode de pensée s'ancre dans le corporel en même temps qu'il émerge du corporel (cerveau compris), ou encore que les processus sensoriels guident et influencent la construction et le fonctionnement de la pensée sur soi, sur autrui et sur le monde, et réciproquement, nous proposons qu'il existe une corrélation logique entre continuum de traitement sensoriel et continuum de mode de pensée. Ainsi, Temple Grandin, qui se définit comme une adulte avec syndrome d'Asperger, décrit son mode de pensée en images, sous formes de séries quasi-infinies de photographies ou de vidéos dynamiques courtes, faisant une place prépondérante à la mémoire (c'est-à-dire des souvenirs stockés et figés). Elle souligne également "Les déficiences mineures du traitement des informations visuelles renforçaient mon attirance pour certains stimuli (j'adorais regarder les portes coulissantes des supermarchés ou des aéroports), qui auraient effrayé ou fait fuir un autre enfant atteint d'anomalies plus sévères ». De cela nous pouvons déduire que des personnes autistes plus sévèrement atteintes, dont les désordres sensoriels portant sur les flux visuels et auditifs seraient plus profonds, auraient une pensée encore plus figée, statique.

Nous avions précédemment proposé que la pensée en images statiques, c'est-à-dire privée de la continuité inhérente au mouvement, pouvait constituer une sorte de signature de l'autisme typique (Gepner, 2001).

Schématiquement, la pensée de personnes atteintes d'autisme sévère fonctionnerait avec un manque très profond de continuité, telle une pensée stroboscopique, une pensée fragmentée, fractionnée, succession chaotique d'images instantanées sans lien entre elles. A mesure que l'on s'éloignerait des formes d'autisme sévère sur le continuum, la pensée acquiérerait une forme de plus en plus dynamique, c'est-à-dire les propriétés d'un flux de plus en plus fluide ${ }^{8}$.

Selon notre hypothèse, il y aurait donc une corrélation entre degré de désordres de traitement temporel des flux sensoriels et de malvoyance é-motionnelle d'un côté, et degré de fluidité et de dynamique de la pensée de l'autre.

\footnotetext{
${ }^{8}$ Chez les personnes ordinaires, «neuro-typiques » (comme les appellent entre elles les personnes présentant un syndrome d'Asperger sur certains sites internet), la pensée courante s'affranchirait plus ou moins complètement des images pour ne fonctionner qu'avec des mots, des symboles, tandis que dans le rêve la pensée retournerait à notre insu, inconsciemment, se retremper dans des modalités visuelles plus archaïques sur le plan développemental.
} 
Le grand philosophe Henri Bergson a écrit dans l'Evolution créatrice (1941) : «Nous prenons des vues quasi-instantanées sur la réalité qui passe, et, comme elles sont caractéristiques de cette réalité, il nous suffit de les enfiler le long d'un devenir abstrait, uniforme, invisible, situé au fond de l'appareil de la connaissance... Perception, intellection, langage procèdent en général ainsi. Qu'il s'agisse de penser le devenir, ou de l'exprimer, ou même de le percevoir, nous ne faisons guère autre chose qu'actionner une espèce de cinématographe intérieur ».

Pour toutes les raisons mentionnées plus haut, nous en sommes venus à penser que de nombreuses personnes autistes ont un problème avec leur cinématographe intérieur, et cette vue nous semble avoir un impact heuristique important sur les relations entre pensée, temps et mouvement. Nous avons déjà esquissé une approche neuropsychodynamique de la pensée en générale et de la pensée autistique en particulier (Gepner, 2001). Selon celle-ci la pensée (sur soi, les autres et le monde) est très profondément inscrite dans le mouvement, elle s'ancre dans le mouvement en même temps qu'elle émerge du mouvement, qu'il s'agisse du mouvement perçu, internalisé, mémorisé, anticipé, agi de manière spontanée, réflexe, puis intentionnalisé.

Un désordre précoce de l'intégration et de l'inscription du mouvement et du temps dans le corps propre entravera le processus développemental de la pensée en mouvement. La Motion (action de mouvoir, mise en mouvement d'un corps physique), la Motilité (faculté de se mouvoir physiquement), la Motion pulsionnelle ${ }^{9}$, tout comme enfin l'Émotion (mouvement de l'âme, mouvement ou transport psychique), procèdent essentiellement d'une même essence. Altéré dans sa capacité à associer et intégrer le mouvement physique et humain, les flux sonore et tactilo-kinesthésique dans son monde intérieur, l'enfant autiste sera aussi perturbé dans le ressenti et l'expression de ses émotions et de sa pensée. Une déformation de la perception de la continuité, co-substantielle au mouvement incessant du monde, aboutira logiquement à une succession d'instants sans lien entre eux.

Dans la même ligne, et pour faire écho à nos hypothèses neurobiologiques de dyssynchronie et dysconnectivité cérébrale au centre des désordres autistiques, comment supposons-nous que la pensée fonctionne lorsque ses substrats neuronaux ne peuvent pas traiter le monde en ligne, et qu'ils fonctionnent de manière dyssynchrone et dysconnectée ? Sera-t-elle capable «d'enfiler les vues quasi-instantanées de la réalité le long d'un devenir abstrait, uniforme, invisible », comme écrit Bergson? Que signifie une conscience temporelle, ou temporospatiale, une conscience de l'espace-temps, de la durée, quand le cerveau ne fonctionne pas à l'heure, en temps réel? On suppose qu'une telle pensée fonctionne en dehors de l'espace-temps ordinaire, dans un espace-temps difficile à imaginer, désynchronisé, discontinu, distordu, morcelé, fragmenté, et sans doute assez effrayant.

Vivant dans cet espace-temps discontinu et disjoint, désuni et morcelé, et essayant d'y survivre, de nombreuses personnes autistes s'auto-stimulent en bougeant le corps ou une partie de celui-ci, car la nature humaine a généralement horreur de l'inertie. Ainsi, ce qui apparaît comme un symptôme typique d'autisme se révèlerait avant tout comme une stratégie compensatoire désespérée de la personne autiste pour se sentir vivante, dynamique, et pour tenter d'inscrire le temps et le mouvement dans son corps propre.

\section{(2) Personnalité autistique ?}

Dans ce paragraphe, nous voudrions poser la question de l'existence d'une personnalité autistique. Est-ce que la personnalité autistique existe ? Est-ce qu'existent des traits de personnalité autistique ? Si tel était le cas, quels seraient les liens quantitatifs et qualitatifs

\footnotetext{
${ }^{9}$ Motion pulsionnelle, triebregung, terme apparenté au mobile, au motif, à la motivation dont Freud dit : "Nous ne pouvons avoir rien d'autre en vue qu'une motion pulsionnelle dont le représentantreprésentation est inconscient", 1915, in Laplanche et Pontalis, 1967).
} 
entre la pathologie autistique et la personnalité autistique ? Dans la constellation autistique, y aurait-il une place pour la personnalité autistique ? Les désordres du spectre autistique sont des désordres neurodéveloppementaux. Sur ce continuum, l'autisme le plus sévère se situe à une extrêmité, le syndrome d'Asperger se situe à l'autre extrêmité, mais au-delà de cette extrêmité, peut-on trouver une condition autistique encore plus légère, qui ne serait pas encore dans le registre de la normalité, mais qu'on pourrait nommer personnalité autistique ?

On peut s'accorder sur le fait que la personnalité est l'identité psychologique subjective d'une personne, faite de propensions, tendances et potentialités, qu'elle est la résultante de facteurs et influences génétiques, épigénétiques et environnementales (éducatives, morales) et enfin qu'elle s'exprime à travers le caractère, les motivations, intentions, désirs et choix conscients et inconscients, mais aussi à travers le contenu du langage, la voix (sa fréquence, son intensité), les mimiques faciales, le langage des yeux, et bien sûr à travers le comportement.

Sur cette toile de fond, que serait la personnalité autistique ? Considérons qu'elle serait au minimum marquée par la solitude, l'isolement ou l'indépendance, des intérêts abstraits, une tendance contemplative et un sens social peu développé. Considérons maintenant un scénario selon lequel un bébé présenterait les caractéristiques d'une telle proto-personnalité dès les débuts de sa vie (pour des raisons génétiques, épigénétiques ou environnementales). Si aucun facteur de risque supplémentaire, aucun événement physique ou psychique majeur ne survient dans la vie de ce bébé, il se peut qu'il grandisse avec cette personnalité autistique. En revanche, si un facteur de risque somatique ou psychique survient, la proto-personnalité de ce bébé pourrait décompenser et évoluer vers l'un ou l'autre des désordres de la constellation autistique. Nous avions préalablement proposé cette approche (Gepner et Soulayrol, 1994), en utilisant les concepts d'épigenèse et d'auto-organisation pour rendre compte de la manière dont le cerveau et le psychisme d'un individu intègrent les facteurs intrinsèques et extrinsèques, et expliquer comment des désordres d'épigenèse et d'auto-organisation conduisent à l'émergence d'un syndrome autistique chez un enfant.

Même si ce scénario psychopathologique est spéculatif, il est néanmoins similaire à celui qui lie un trouble de la personnalité avec une maladie mentale, par exemple la personnalité schizoïde avec la schizophrénie, la personnalité obsessionnelle avec un trouble (ou une névrose) obsessif-compulsif, ou la personnalité paranoïaque avec la psychose paranoïaque. De plus il nous permet de rendre compte de la réalité clinique et psychopathologique subtile et quasi-infinie des désordres de la constellation autistique.

\section{(3) Dissociation psychisme-cerveau dans l'autisme?}

Le grand neuro-psychiatre Henri Baruk, faisant référence à Babinski dont il fut l'élève, qui avait souligné que toutes les atteintes organiques ou fonctionnelles des centres du système nerveux et du cerveau se traduisent uniquement par des manifestations dissociées, a écrit en 1985 : "Une longue observation des malades mentaux nous a montré que même chez des malades délirants, incohérents ou incompréhensibles, persiste, si on sait avoir leur confiance et les considérer avec sympathie, une personnalité profonde, sensible, qui a perdu les moyens logiques de s'exprimer, mais qui reste bien vivante, qui vibre et qui souffre...Cette personnalité profonde que nous avons longuement étudiée dans notre ouvrage de Psychiatrie morale (1945), c'est l'âme du malade ».

En quoi consiste l'autisme, cette condition de vie particulière, révélée par un certain degré de dissociation entre soi et les autres ? Selon notre approche neuropsychologique d'un désordre du traitement temporospatial des flux sensoriels avec malvoyance é-motionnelle, sous-tendue neurobiologiquement par une dyssynchronie et dysconnectivité neuronale multisystème, les personnes avec autisme présentent au moins les désordres suivants : dyssynchronisation des événements sensoriels au sein d'une modalité sensorielle et entre plusieurs modalités sensorielles, déficit de cohérence temporelle, découplage sensorimoteur, désordres de 
l'anticipation motrice, désordres de l'imitation, désordres de compréhension verbale et émotionnelle, désordres attentionnels, ainsi que des symptômes révélant un certain degré de compensation sensorielle et sensori-motrice.

En somme, la perception cohérente du monde par simultanéité et la conscience de soi, des autres et du monde par synchronisation, sont compromises à un certain degré dans l'autisme. En suivant cette ligne, nous avons proposé qu'il existe une dissociation entre psychisme et cerveau-corps dans l'autisme (Gepner, 2003; voir aussi François, 1997). Dans quel sens ?

A travers la Communication Facilitée (une méthode contoversée utilisée dans différents handicaps physiques et mentaux à travers le monde), Pierre, un adulte autiste a écrit :

"J'avais un gros problème de synchronisation et de commande du geste. J'étais comme paralysé. Serrer la main était très difficile. Je composais intérieurement le geste. Je l'envoyais image par image comme dans un film muet. Je percevais mes gestes comme saccadés, comme des images manquantes qui n'arrivaient pas assez vite. Il y avait parfois un très long temps entre le moment où j'avais l'intention de faire un geste et celui où j'arrivais à le réaliser. L'impulsion mentale voulait faire le geste, mon corps se mettait à vibrer, comme un picotement électrique, et tout se bloquait. Le schéma corporel ne suivait pas ma volonté. J'avais l'impression de pousser un mur. Je parlais à mon corps comme à une personne étrangère » (in Vexiau, 1996).

Un autre patient autiste, Birger Sellin (1998) écrit : «... je dirige mon moi intérieur sans pouvoir influer sur ma conduite élémentaire, cette incapacité me paraît être un problème majeur de l'autisme... je sais comment agissent les phrases les mots comment on les entend intérieurement mais à l'extérieur c'est différent sans contrôle des mondes intérieurs... ».

Chez ces patients, la pensée et les affects (que Freud nommait de façon peut-être prémonitoire les «quanta d'affects»), qui s'expriment habituellement à travers le langage, le corps et les émotions, fonctionnent sans pouvoir résolutif sur le cerveau et le corps langagier et émotionnel. Du coup, le cerveau fonctionne de manière plus ou moins autonome, dissociée, déliée, désynchronisée par rapport à la pensée et aux affects. Selon cette perspective, l'autisme procéderait de désordres de la liaison entre leur attention, leur intention, leur volition, leur conscience, d'une part, et leur cerveau et leur corps d'autre part. L'unité psychosomatique se serait mal construite, mal unifiée.

On ne peut que remarquer les similarités entre ces descriptions de patients autistes et ce que des patients atteints de la maladie de Parkinson pourraient dire de leur extrême difficulté à contrôler leur corps avec leur esprit, de connecter leur idéation et leur volition à leur action. De fait, plusieurs travaux confirment la parenté entre certains symptômes moteurs dans ces deux pathologies neuro-psychiques (Vilensky et al., 1981 ; Maurer \& Damasio, 1982 ; Jones \& Prior, 1985 ; Kohen-Raz et al., 1992 ; Hallet et al., 1993; Leary \& Hill, 1996, pour une revue).

Qu'est-ce d'autre qui caractérise la dissociation psychisme-cerveau dans l'autisme ?

Comme nous l'avons vu dans les paragraphes précédents, le monde va probablement trop vite pour certaines personnes autistes. Dès lors, les personnes autistes ont une conscience différente de la durée, et ont besoin de davantage de temps pour négocier avec le monde. "Le changement perpétuel qu'il fallait affronter partout ne me donnait jamais le temps de me préparer... la tension qu'exigeait la nécessité d'attraper les choses au vol pour se les assimiler fut le plus souvent trop forte pour moi. Il me fallut trouver un biais pour ralentir les choses afin de m'accorder le temps de négocier avec elles... », écrit Williams (1992). Dans ce contexte, la théorie fictive proposée par Philip K. Dick (1964) dans son Glissement de temps sur Mars était sans doute prémonitoire. Certaines personnes autistes n'ont probablement que peu ou pas de conscience de la durée. Le temps est probablement vécu de manière plus ou moins fractionnée, comme des instants erratiques sans durée ni continuité, et l'espace est vécu comme un puzzle d'images statiques peu ou pas liées entre elles. Et si certaines personnes autistes réussissent, et parfois excellent, à rassembler les pièces du puzzle, et si d'autres produisent de la continuité ainsi qu'une forme d'auto-réassurance et parfois d'auto-rééducation à travers leurs stéréotypes 
verbaux et moteurs, la plupart continuent de vivre dans leur monde privé plus ou moins ouvert aux autres.

Cette hypothèse de dissociation psychisme-cerveau-corps dans l'autisme suppose une augmentation de l'autonomie relative du psychisme et de la pensée par rapport au cerveaucorps (Gepner, 2003). Selon nous, l'esprit et le corps des personnes autistes fonctionnent dans des dimensions relativement séparées, avec insuffisamment d'influences réciproques entre eux. Le psychisme intègre et contrôle insuffisamment les activités cérébrales et corporelles, et peut ainsi fonctionner dans un monde plus ou moins abstrait. "Est-ce pour avoir été absorbée dans les taches et les étoiles au moment précis où les autres enfants s'ouvrent au monde extérieur que je suis restée sur le bord de la route? » se demande Donna Williams.

\section{(4) Relations psychisme-cerveau et dualisme interactionniste}

Si cette vue a quelque réalité, elle a beaucoup à voir avec les travaux de Sir John Eccles (prix Nobel de médecine en 1963) et sa théorie du dualisme interactionniste (Eccles, 1951, 1994). Selon ce savant relativement oublié, la possibilité existe que l'esprit et le cerveau interagissent à travers des processus quantiques, et en particulier que l'intention et l'attention, considérées par Eccles et Popper comme des événements psychiques conscients immatériels, accroissent la probabilité d'exocytose d'une vésicule synaptique dans l'espace inter-synaptique. En tant que telles, l'intention et l'attention contrôlent, ou à tout le moins agissent sur, le cerveau, par le biais d'un recrutement neuronal de grande ampleur (voir Gepner, 2003 pour une réflexion critique sur ce modèle).

Une découverte extrêmement importante selon nous est venue créditer cette approche : Lutz et al. (2004) ont démontré que des moines bouddhistes méditants présentent une augmentation très significative de l'activité gamma de synchronisation cérébrale pendant qu'ils méditent, par rapport à des sujets témoins ordinaires. Autrement dit, une activité mentale volontaire particulière (dans l'expérience de Lutz et al., les moines méditent sur la compassion) influence le fonctionnement cérébral. Des auteurs comme Varela avaient déjà montré qu'un acte cognitif tel que la perception consciente d'une forme est corrélé à une augmentation de l'activité de synchronisation gamma chez l'adulte normal (Rodriguez et al., 1999). Mais ce que Lutz et al. démontrent est la première corrélation claire, à notre connaissance, entre d'une part une activité mentale complexe hautement conscientisée, la méditation, elle-même liée à un haut degré d'attention, d'intentionnalité, de concentration et de motivation, et d'autre part un haut degré de synchronisation cérébrale. De plus, les auteurs montrent une corrélation entre le nombre d'heures de pratique méditative et l'intensité de la synchronisation cérébrale. Ce résultat est le premier argument quantitatif et qualitatif majeur en faveur de l'influence d'une activité mentale ou psychique consciente sur le cerveau, et ouvre selon nous la voie à un champ immense de possibilités théorico-cliniques.

Il ne s'agit évidemment pas d'écarter les influences réciproques de l'activité cérébrale sur la pensée et le psychisme à travers les concepts puissants d'auto-organisation et d'émergence mais qui, en dépit d'une abondante littérature scientifique et philosophique (à laquelle nous avons nous-même contribué, Gepner et Soulayrol, 1994), restent encore largement spéculatifs. Mais en poursuivant cette voie théorique et expérimentale ouverte il y a un demi-siècle par Eccles (1951), nous proposons de considérer attentivement son dualisme interactionniste comme une alternative plausible à l'interprétation moniste-matérialiste contemporaine actuellement dominante, selon laquelle la conscience, l'esprit, serait une sorte de création ou de sous-produit du cerveau. En postulant des effets quantiques au cœur du fonctionnement du cerveau (Beck \& Eccles, 1992), cette approche remet le dualisme cartésien sur l'agenda des neurosciences et de la physique modernes. A côté des trois dimensions de l'espace et de celle du temps, qui gouvernent en partie le fonctionnement de la matière, cerveau compris, nous 
postulons l'existence d'une «cinquième dimension", subtile, purement psychique, mais relative aux 4 dimensions de l'espace-temps. Nous proposons que le psychisme et le cerveau fonctionnent à/dans des degrés de matérialité distincts ${ }^{10}$, qu'ils sont étroitement et logiquement compatibles entre eux le temps d'une vie humaine, deviennent quasiment indistincts l'un de l'autre en cas de fonctionnement neuro-psychique ordinaire, mais se dissocient et fonctionnent de manière relativement autonome l'une par rapport à l'autre en cas de maladies neuro-psychiques (Gepner, 2003). Dans l'autisme, la dyssynchronie neuronale conduit à une perception temporelle du monde altérée. Réciproquement, les idées, sentiments et intentions ne peuvent être convertis de manière adéquate en activité cérébrale.

\section{Conclusion}

A côté des termes très pertinents de spectre ou continuum autistique, nous voudrions ajouter le terme imagé de constellation autistique pour continuer à rendre (mieux ?) compte de plusieurs réalités relatives à ces désordres : d'une part, le terme constellation évoque un nuage constitué d'éléments singuliers, qui symboliseraient peut-être mieux les personnes atteintes de ces désordres que des tableaux cliniques sur un axe nosographique ; constellation évoque aussi une distribution des désordres autistiques dans un espace à trois dimensions (et non plus deux comme dans un spectre ou un continuum), auxquelles s'ajoute la dimension du temps, puisque les constellations sont en extension ou en mouvement perpétuels, et nous avons tenté de montrer à quel point le mouvement et le temps étaient importants pour aborder ces désordres ; constellation suppose aussi des rapprochements ou éloignements de ces éléments entre eux, certains éléments pouvant passer d'une constellation à une autre; aussi bien, nous avons vu que des recouvrements nosographiques existaient entre désordres autistiques et autres désordres neurodéveloppementaux. Nous arrêterons là les analogies avant de spéculer sur une origine commune à ces désordres de la constellation autistique. Car comme nous l'avons rappelé en introduction, il n'y a pas une origine commune à ces désordres.

En revanche, nous avons proposé dans cet article l'idée qu'un processus de synchronisationconnectivité neuronale était probablement affecté dans l'ensemble de ces désordres, de même que dans d'autres désordres neurodéveloppementaux et neuropsychiques. Aussi différents soient-ils sur le plan clinique, les différents troubles neurodéveloppementaux, ou les différentes maladies neuro-psychiques, pourraient en partie comporter dans leurs soubassements neurobiologiques fondamentaux, une altération de la synchronisationconnectivité neuronale. Cette altération, en fonction de ses origines, de sa nature, de son intensité, de ses localisations, de son extension topologique et temporelle (temporo-spatiale), se manifesterait par des expressions phénotypiques variées, c'est-à-dire différentes constellations de désordres neuropsychiques. Cette approche neurobiologique replace et relativise l'autisme au sein de la pathologie neurodéveloppementale et neuropsychique.

Nous avons argumenté l'hypothèse de défauts d'intégration temporospatiale des flux sensoriels dans l'autisme, outre celle de la Malvoyance é-motionnelle, en montrant que les personnes autistes avaient de plus ou moins grandes difficultés à inscrire les mouvements

\footnotetext{
10 On sait, depuis les travaux de Rizzolatti et al. (1996), qu'imaginer un événement, le voir et l'agir, partagent des processus neuraux communs, et que voir l'événement sollicite plus de groupes neuronaux que seulement l'imaginer, et que l'agir en sollicite davantage que le percevoir. Il existe un continuum énergétique entre pensée, perception et action. En d'autres termes, ce qui différencie ces différentes activités de l'esprit-cerveau humain est le degré d'activité mentale et neuronale, c'est-à-dire le degré d'énergie mise dans le processus, avec un accroissement de recrutement neuronal et de synchronisation neuronale entre le processus d'imagination (d'idéation pure), celui de la perception, et celui de l'action, c'est-à-dire un accroissement de degré d'énergie et de matière impliqués dans le processus.
} 
physique et humain, tels que l'émotion faciale, mais aussi le flux de la parole et le flux tactile, et enfin le temps et la durée dans leur corps propre et leur pensée. Cette inscription, au lieu de se faire de manière continue, synchronisée, cohérente, se ferait de manière fragmentée, désynchronisée et à retardement. Vivant dans un monde trop rapide et changeant pour lui, un monde aux contraintes temporospatiales trop élevées pour lui et différentes des nôtres, l'enfant autiste aurait des difficultés à se lier et s'accorder en temps réel et de manière adaptée avec le monde physique et humain. Certains enfants autistes parviennent à corriger en partie leur perception discontinue, désunie et incohérente du monde environnemental, en le ralentissant, en le figeant, en le répétant, ou à compenser leurs troubles perceptifs en surinvestissant des singularités ou des détails d'événements sensoriels.

Nous pensons que notre approche neuropsychologique développementale confirme les concepts psychanalytiques post-kleiniens de démantèlement perceptif, d'identification adhésive, et de bi-dimensionnalité, c'est-à-dire d'un monde privé de troisième dimension constitutive d'un espace psychique, et de quatrième dimension temporelle. Comme l'avait proposé Meltzer et al. (1980), l'évolution d'un enfant autiste serait marquée par l'accès à une troisième puis une quatrième dimension.

Nous avons également présenté les premiers arguments démontrant l'utilité de ralentir le monde environnant pour faciliter la perception et l'intégration du monde environnant par les enfants autistes (Lainé et al;, sous presse; Tardif et al., in press). Nous testons actuellement un logiciel destiné à ralentir la présentation aux enfants autistes des mouvements et de la parole d'un interlocuteur, et en mesurons les effets sur leurs capacités imitatives et de compréhension du langage. Nos premiers résultats sont encourageants.

Nous avons proposé dans la dernière partie de considérer l'autisme comme un paradigme de dissociation entre psychisme et cerveau. De récentes découvertes viennent de confirmer l'idée restée jusque-là théorique chez les neuroscientifiques, d'une autonomie relative du psychisme conscient par rapport au cerveau, et d'une influence du premier sur le second par l'intermédiaire de la synchronisation, de la connectivité et de la plasticité neuronale, une réalité déjà postulée il y a un siècle par Freud avec son concept d'inconscient et sa description de ses effets sur le corps. Nous pensons que notre approche dualiste-interactionniste des relations entre psychisme et cerveau (et corps), située à l'interface des événements neurobiologiques et psychiques, réintroduit le sujet, l'observateur, le témoin, le clinicien, et le chercheur, avec toute sa subjectivité, sa singularité, et son libre-arbitre, au coeur des neurosciences et des sciences du psychisme, et qu'elle est susceptible de créer de fécondes passerelles entre elles.

Il nous reste à clore cet article en souhaitant que notre nouvelle approche puisse à l'avenir ouvrir des possibilités théoriques, expérimentales et cliniques pour mieux penser, soigner, et communiquer. 


\section{Références bibliographiques}

Adolphs, R., Tranel, D., Damasio, H., \& Damasio, A. (1994). Impaired recognition of emotion in facial expressions following bilateral damage to the human amygdala. Nature, 372, 669-672.

Allison T., Puce A, McCarthy G (2000). Social perception from visual cues: role of the STS region. Trends in Cognitive Sciences, 4, 267-278.

American Psychiatric Association (1994). Diagnostic and Statistical Manual of Mental Disorders (DSM-IV), 4th ed. Washington DC: APA.

Babiloni, C., Bares, M., Vecchio, F., Brazdil, M., Jurak, P., Moretti, D.V., Ubaldi, A., Rossini, P.M., \& Rektor, I. (2004). Synchronization of gamma oscillations increases functional connectivity of human hippocampus and inferior-middle temporal cortex during repetitive visuomotor events. European Journal of Neuroscience, 19, 3088-3098.

Bailey, A., Luthert, P., Dean, A.,Harding, B., Janota, I., Montgomery, M., Rutter, M., \& Lantos, P. (1998). A clinicopathological study of autism. Brain, 121, 889-905.

Baron-Cohen, S., Campbell, R., Karmiloff-Smith, A., \& Grant, J. (1995). Are children with autism blind to the mentalistic significance of the eyes ? British Journal of Devevelopmental Psychology, 13, 379-398.

Baron-Cohen, S., Ring, H., Bullmore, E., Wheelwright, S., Ashwin, C., \& Williams, S. (2000). The amygdala theory of autism. Neuroscience Biobehavioral Review, 24, 355-364.

Baruk, H. (1985). Essai sur la médecine hébrä̈que dans le cadre de l’histoire juive. Paris : Colbo.

Beck, F. \& Eccles, J.C. (1992). Quantum aspects of brain activity and the role of consciousness. Proceedings of the National Academy of Science, 89, 11357-11361.

Belin, P., Zatorre, R.J., Lafaille, P., Ahad, P., Pike, B. (2000). Voice-selective areas in human auditory cortex. Nature, 403, 309-312.

Belmonte, M.K., Allen, G., Beckel-Mitchener, A., Boulanger, L.M., Carper, R.A., \& Webb, S.J. (2004). Autism and abnormal development of brain connectivity. Journal of Neuroscience, 24, 9228-9231.

Bergson, H. (1941). L'évolution créatrice. Paris : Presses Universitaires de France.

Berthoz A (1997). Le sens du mouvement. Paris : Odile Jacob.

Bertone A., Mottron L., Jelenic P, Faubert J. (2003). Motion perception in autism: a 'complex' issue. Journal of Cognitive Neuroscience, 15, 218-225.

Blake R., Turner L.M., Smoski M.J., Pozdol S.L. \& Stone W.L. (2003). Visual recognition of biological motion is impaired in children with autism. Psychological Science, 14, 151-157.

Boisacq-Schepens, N. \& Crommelinck, M. (1994). Neuro-psycho-physiologie, vol. 1, Fonctions sensorimotrices, Paris : Masson.

Borgers, C., \& Kopell, N. (2003). Synchronization in networks of excitatory and inhibitory neurons with sparse, random connectivity. Neural Computers, 15, 509-538.

Boucher, J., \& Lewis, V. (1992). Unfamiliar face recognition in relatively able autistic children. Journal of Child Psychology and Psychiatry, 33, 843-859.

Bourguignon, A. (1981). Fondements neurobiologiques pour une théorie de la psychopathologie, un nouveau modèle. La Psychiatrie de l'Enfant, XXIV, 2, 445-540.

Breakspear, M. (2004). "Dynamic" connectivity in neural systems: theoretical and empirical considerations. Neuroinformatics, 2, 205-226. 
Brock, J., Brown, C.C., Boucher, J. \& Rippon, G. (2002). The temporal binding deficit hypothesis of autism. Developmental Psychopathology, 14, 209-224.

Brown, C., Gruber, T., Boucher, J., Rippon, G., \& Brock, J. (2005). Gamma abnormalities during perception of illusory figures in autism. Cortex, 41, 364-376.

Butterworth, G \& Hicks, L. (1977). Visual proprioception and postural stability in infancy. A developmental study. Perception, 6, 255-262.

Castelli F., Frith C., Happé F., Frith U. (2002). Autism, Asperger syndrome and brain mechanisms for the attribution of mental states to animated shapes. Brain, 125, 1839-1849.

Celani, G., Battacchi, M.W., \& Arcidiacono, L. (1999). The understanding of the emotional meaning of facial expressions in people with autism. Journal of Autism and Developmental Disorders, 29,57-66.

Changeux, J-P. (1983). L'homme neuronal. Paris : Fayard.

Cohen, I.L. (1994). An artificial neural network analogue of learning in autism. Biological Psychiatry, 36, 5-20.

Courchesne E (1997). Brainstem, cerebellar and limbic neuroanatomical abnormalities in autism. Current Opinion in Neurobiology, 7, 269-278.

Courchesne, E., Saitoh, O., Yeung-Courchesne, R., Press, G.A., Lincoln, A.J., Haas, R.H., \& Schreibman, L. (1994). Abnormality of cerebellar vermian lobules VI and VII in patients with infantile autism: identification of hypoplastic and hyperplastic subgroups with MR imaging. American Journal of Roentgenology, 162 2 123-130.

Courchesne, E. \& Pierce, K. (2005). Why the frontal cortex in autism might be talking only to itself: local overconnectivity but long-distance disconnection. Current Opinion in Neurobiology, 15, 225-230.

Critchley, H.D., Daly, E.M., Bullmore, E.T., Williams, S.C., Van Amelsvoort, T., Robertson, D.M., Rowe, A., Phillips, M., Mcalonan, G., Howlin, P., \& Murphy, D.G. (2000). The functional neuroanatomy of social behaviour: changes in cerebral blood flow when people with autistic disorder process facial expressions. Brain, $123,2203-2212$.

Davies, S., Bishop, D., Manstead, A.S.R., \& Tantam, D. (1994). Face perception in children with autism and Asperger's syndrome. Journal of Child Psychology and Psychiatry, 35, 1033-1057.

Dawson, G., Webb, S.J., \& Mc Partland, J. (2005). Understanding the nature of face processing impairment in autism: insights from behavioral and electrophysiological studies. Developmental Neuropsychology, 27, 403424.

De Gelder, B., Vroomen, J., \& Van der Heide, L. (1991). Face recognition and lip-reading in autism. European Journal of Cognitive Psychology, 3, 69-86.

Deruelle, C., Rondan, C., Gepner, B., Tardif, C. (2004). Spatial frequency and face processing in children with autism and Asperger syndrome. Journal of Autism and Developmental Disorders, 34, 199-210.

Deruelle C, Rondan C, Gepner B, Fagot J. (2006). Processing of compound visual stimuli by children with autism and Asperger syndrome. International Journal of Psychology, 41, 97-106.

Dick, P.K. (1964). Martian time-sleep. New York: Ballantine. French translation: Glissement du temps sur Mars, Presses de la cité, 1994, p. 276-277.

Doya, K. (2000). Complementary roles of basal ganglia and cerebellum on learning and motor control. Current Opinion in Neurobiology, 10, 732-739.

Eccles, J.C. (1951). Hypotheses relating to the brain-mind problem. Nature, 4263, 53-57. 
Eccles, J.C. (1994). How the self controls its brain. Trad. française "Comment la conscience contrôle le cerveau », Paris : Fayard, 1997.

François, G. (1997). L'autisme en questions. Paris : Buchet Chastel.

Freud, S. (1915). Das umbewusste, 1915, cité in Laplanche \& Pontalis, Vocabulaire de la psychanalyse, Paris : P.U.F., 1967.

Frith U. (1989). Autism : explaining the enigma. Basic Blackwell.

Gepner, B., Buttin, C., de Schonen, S. (1994). Face processing in young autistic children. Infant Behavior and Development, 17, 661.

Gepner, B. \& Soulayrol, R. (1994). Utilité des concepts d'épigenèse et d'auto-organisation pour la compréhension des syndromes autistiques de l'enfant. La Psychiatrie de l'Enfant, XXXVII, 1, 115-152.

Gepner, B., Mestre, D., Masson, G., de Schonen, S. (1995). Postural effects of motion vision in young autistic children. NeuroReport, 6, 1211-1214.

Gepner, B., de Gelder, B. \& de Schonen, S. (1996). Face processing in autistics : Evidence for a generalized deficit? Child Neuropsychology, 2, 123-139.

Gepner B. (1997). Reconnaissance des visages et perception visuelle du mouvement chez les enfants autistes. Thèse de doctorat de neurosciences. Université Aix-Marseille II.

Gepner B. (2001). «Malvoyance» du mouvement dans l'autisme infantile ? Une nouvelle approche neuropsychopathologique développementale. La Psychiatrie de l'Enfant, 1, 77-126.

Gepner B., Deruelle C., Grynfeltt S. (2001). Motion and emotion: a novel approach to the study of face processing by autistic children. Journal of Autism and Developmental Disorders, 31, 37-45.

Gepner B. \& Massion J. (dirigé par) (2002). L'autisme : une pathologie du codage temporel ? Revue TIPA (Revue des Travaux Interdisciplinaire du laboratoire PArole et langage, 21, 177-218.

Gepner B. \& Mestre D. (2002a). Postural reactivity to fast visual motion differentiates autistic from children with Asperger syndrome. Journal of Autism and Developmental Disorders, 32, 231-238.

Gepner B. \& Mestre D. (2002b). Rapid visual-motion integration deficit in autism. Trends in Cognitive Sciences, $6,11,455$.

Gepner, B. (2003). Relations psychisme-cerveau, dualisme interactionniste et gradients de matérialité. Intellectica (Revue de l'Association pour la Recherche Cognitive), 36-37, 319-340.

Gepner, B. (2004). Autism, movement and facial processing. The American Journal of Psychiatry, 161, 1719.

Gepner, B. (2005). Malvoyance du mouvement dans l'autisme: de la clinique à la recherche et à la rééducation. L'Autisme : de la recherche à la pratique, C. Andrès, C. Barthélémy, A. Berthoz, J. Massion, B. Rogé (Eds.), p. 205-226, Paris , Odile Jacob.

Gepner, B., Lainé, F., Tardif, C. (2005). E-Motion mis-sight and other temporal processing disorders in autism. Current Psychology of Cognition/Cahiers de Psychologie Cognitive, 23, 104-121.

Gepner, B. et Tardif, C. (in press). Autism, movement, time and thought. In F. Colombus (Ed.) Frontiers in Cognitive Psychology. New York : Nova Science publishers.

Gervais, H., Belin, P., Boddaert, N. et al. (2004). Abnormal cortical voice processing in autism. Nature Neuroscience, 7, 801-2.

Gibson J.J. (1979). The ecological approach to visual perception. Boston, Houghton Mifflin. 
Grandin T. (1995). Thinking in pictures and other reports from my life with autism, Doubleday.

Habib M., Espesser R., Rey V., Giraud K., Bruas P., Gres C. (1999). Training dyslexics with acoustically modified speech : evidence of improved phonological performance. Brain and Cognition, 40, 143-46.

Habib, M. (en préparation). Troubles associés et comorbidités dans la dyslexie: de l'observation clinique à la compréhension des mécanismes. In Expertise collective sur les Troubles de Développement du Langage oral et écrit, Institut National de la Santé et de la Recherche Médicale, Editions INSERM : Paris).

Hadjikhani, N., Joseph, R.M., Snyder, J., Chabris, C.F., Clark, J., Steele, S., McGrath, L., Vangel, M., Aharon, I., Feczko, E., Harris, G.J., \& Tager-Flusberg, H. (2004). Activation of the fusiform gyrus when individuals with autism spectrum disorder view faces. Neuroimage, 22, 1141-1150.

Happé F. (1999). Autism: Cognitive deficit or cognitive style? Trends in Cognitive Sciences, 3, 216-222.

Hallett, M., Lebiedowska, M.K., Thomas, S.L., Stanhope, S.J., Denckla, M.B. \& Rumsey, J. (1993). Locomotion of autistic adults. Archives of Neurology, 50, 1304-1308.

Haxby, J.V., Gobbini, M.I., Furey, M.L., Ishai, A., Schouten, J.L., \& Pietrini, P. (2001). Distributed and overlaping representations of faces and objects in ventral temporal cortex. Science, 293, 2425-2430.

Hertzig, M.E., Snow, M.E,. \& Sherman, M. (1989). Affect and cognition in autism. Journal of the Amercican Academy of Child and Adolescent Psychiatry, 28, 195-199.

Hobson R.P. (1986, b). The autistic child's appraisal of expressions of emotion: a further study. Journal of Child Psychology and Psychiatry, 27, 671-680.

Hobson, R.P. (1986, a). The autistic child's appraisal of expressions of emotion. Journal of Child Psychology and Psychiatry, 27, 321-342.

Hobson, R.P., Ouston, J., \& Lee, A. (1988). What's in a face? The case of autism. British Journal of Psychology, $79,441-453$.

Horwitz, B., Rumsey, J.M., Grady, C.L., \& Rapoport, S.I. (1988). The cerebral metabolic landscape in autism. Intercorrelations of regional glucose utilization. Archives of Neurology, 45, 749-755.

Hughes, J.R., \& Melyn, M. (2005). EEG and seizures in autistic children and adolescents: further findings with therapeutic implications. Clinical EEG and Neuroscience, 36, 15-20.

Ito M, (1984). The cerebellum and neural control. Raven Press :New York.

Jamain, S., Quach, H., Betancur, C., Rastam, M., Colineaux, C., Gillberg, I.C., Soderstrom, H., Giros, B., Leboyer, M., Gillberg, C., Bourgeron, T. et al. (2003). Mutations of the X-linked genes encoding neuroligins NLGN3 and NLGN4 are associated with autism. Nature Genetics, 34, $27-29$.

Johnson M.T. et Ebner T.J. (2000.) Processing of multiple kinematic signals in the cerebellum and motor cortices. Brain Research Review, 33, 155-168.

Jones V. and Prior M. (1985). Motor imitation abilities and neurological signs in autistic children. Journal of Autism and Developmental Disorders, 15, 37-46.

Jouen F. (1988). Visual proprioceptive control of posture in newborn. In: Amblard B, Berthoz A and Clarac F, eds. Posture and gait: development adaptation and modulation. Amsterdam: Elsevier, 13-22.

Just, M.A., Cherkassky, V.L., Keller, T.A., \& Minshew, N.J. (2004). Cortical activation and synchronization during sentence comprehension in high-functioning autism: evidence of underconnectivity. Brain, 127, 18111821.

Kanner L (1943). Autistic disturbances of affective contact. Nervous Child, 2, 217-250. 
Kemner, C., van der Geest, J.N., Verbaten, M.N., van Engeland, H. (2004). In search of neurophysiological markers of pervasive developmental disorders : smooth pursuit eye movements? Journal of Neural Tansmission, $111,1617-26$

Kemper, T.L., \& Bauman, M.L. (1993). The contribution of neuropathologic studies to understanding of autism. Neurological Clinics, 11,175-187.

Klin, A., Sparrow, S.S., de Bildt, A., Cicchetti, D.V., Cohen, D.J., \& Volkmar, F.R. (1999). A normed study of face recognition in autism and related disorders. Journal of Autism and Developmental Disorders, 29, 497-507.

Kohen-Raz R., Volkmar F.R., Cohen D.J. (1992). Postural control in children with autism. Journal of Autism and Developmental Disorders, 22, 419-432.

Lainé, F., Tardif, C., Gepner, B. (sous presse). Amélioration de la reconnaissance et de l'imitation d'expressions faciales chez des enfants autistes grâce à une présentation visuelle et sonore ralentie. Annales MédicoPsychologiques.

Langdell, T. (1978). Recognition of faces: an approach to the study of auism. Journal of Child Psychology and Psychiatry, 19, 255-268.

Leary M.R. \& Hill D.A. (1996). Moving on: autism and movement disturbance. Mental Retardation, 1, 39-53.

Lee, D.N. \& Aronson, E. (1974). Visual proprioceptive control of standing in human infants. Perception and Psychophysics, 15, 529-532.

Lelord, G. (1998). L'exploration de l'autisme. Paris : Grasset.

Lewis, R.F. and Zee, D.S. (1993). Ocular motor disorders associated with cerebellar lesions : pathophysiology and topical localization. Revue Neurologique (Paris), 149, 665-677.

Livingstone M. \& Hubel D. (1988). Segregation of form, color, movement and depth: anatomy, physiology and perception. Science, 240, 740-749.

Loveland, K.A., Tunali-Kotoski, B., Pearson, D.A., Brelsford, K.A., Ortegon, J., \& Chen, R. (1994). Imitation and expression of facial affect in autism. Development and Psychopathology, 6, 433-444.

Lutz, A., Greischar, L.L., Rawlings, N.B., Ricard, M. \& Davidson, R.J. (2004). Long-term meditators selfinduce high-amplitude gamma synchrony during mental practice. Proceedings of the National Academy of Sciences, 101, 16369-16373.

Martineau, J., Roux, S., Adrien, J.L., Garreau, B., Barthélémy, C., \& Lelord, G. (1992). Electrophysiological evidence of different abilities to form cross-modal associations in children with autistic behaviors. Electroencephalography and Clinical Neurophysiology, 82, 60-66.

Massion, J. (1997). Cerveau et motricité. Paris, PUF, "Pratiques corporelles".

Massion, J. (1993) Grandes relations anatomo-fonctionnelles dans le cervelet. Revue Neurologique, 11, 600-606.

Maurer, R.G. \& Damasio, A.R. (1982). Childhood autism from the point of view of behavioral neurology. Journal of Autism and Developmental Disorders, 12, 195-205.

Meltzer, D., Brenner, J., Hoxter, S. et al. (1980). Explorations dans le monde de l'autisme, Paris : Payot.

Merzenich, M.M., Jenkins, W.M., Johnston, P., Schreiner, C., Miller, S.L, Tallal, P. (1996). Temporal processing deficits of language-learning impaired children ameliorated by training, Science, 271, 27-28.

Mestre, D., Rondan, C., Masson, G., Castet, E., Deruelle, C., Gepner, B. (2002). Evaluation de la vision du mouvement chez des enfants autistes au moyen du nystagmus opto-cinétique. Revue TIPA, 21, 192-198. 
Middleton, F.A. \& Strick, P.L. (2000). Basal ganglia and cerebellum loops: motor and cognitive circuits. Brain Research Review, 31, 236-250.

Milne, E., Swettenham, J., Hansen, P., Campbell, R., Jeffries, H., Plaisted, K. (2002). High motion coherence thresholds in children with autism. Journal of Child Psychology and Psychiatry, 43, 255-263.

Milne, E., Swettenham, J., Campbell, R. (2005). Motion perception and autistic spectrum disorder: a review. Current Psychology of Cognition/Cahiers de Psychologie Cognitive, 23, 3-33.

Mottron, L. (2004). L'autisme: une autre intelligence. Sprimont: Mardaga.

Nishitani, N., Avikainen, S., \& Hari, R. (2004). Abnormal imitation-related cortical activation sequences in Asperger's syndrome. Annals of Neurology, 55, 558-562.

Oberman, L.M., Hubbard, E.M., McCleery, J.P., Altschuler, E.L., Ramachandran, V.S., \& Pineda, J.A. (2005). EEG evidence for mirror neuron dysfunction in autism spectrum disorders. Brain Research Cognitive Brain Research, 24, 190-198.

Ornitz, E.M. (1974). The modulation of sensory input and motor output in autistic children. Journal of Autism and Childhood Schizophrenia, 4, 197-215.

Ornitz, E.M. \& Ritvo, E.R. (1968). Perceptual inconstancy in early infantile autism. Archives of General Psychiatry, 18, 76-98.

Pellicano, E., Gibson, L., Maybery, M., Durkin, K., Badcock, D.R. (2005). Abnormal global processing along the dorsal visual pathway in autism: a possible mechanism for weak visuospatial coherence ? Neuropsychologia, 43, 1044-53.

Percha, B., Dzakpasu, R., Zochowski, M., \& Parent, J. (2005). Transition from local to global phase synchrony in small world neural network and its possible implications for epilepsy. Physical Review and Statistical Nonlinear Soft Matter Physics, 72, 031909.

Pierce, K., Haist, F., Sedaghat, F., \& Courchesne, E. (2004). The brain response to personally familiar faces in autism: findings of fusiform activity and beyond. Brain, 127, 2703-2716.

Rapin I. (2002). The autistic spectrum disorders. The New England Journal of Medicine, 347, 302-303.

Receveur, C., Lenoir, P., Desombre, H., Roux, S., Barthélémy, C., \& Malvy, J. (2005). Interaction and imitation deficits from infancy to 4 years of age in children with autism : a pilot study based on videotapes. Autism, 9, 6982.

Rizzolatti, G., Fadiga, L., Gallese, V. \& Fogassi, L. (1996). Premotor cortex and the recognition of motor actions. Brain Research. Cognitive Brain Research, 3, 131-141.

Rodriguez, E., George, N., Lachaux, J.P., Martinerie, J., Renault, B. \& Varela, F. (1999). Perception's shadow : long-distance synchronization of human brain activity. Nature, 397, 430-433.

Roessner, V., Banaschewski, T., Uebel, H., Becker, A., \& Rothenberger, A. (2004). Neuronal network models of ADHD-lateralization with respect to interhemispheric connectivity reconsidered. European Child and Adolescent Psychiatry, 13, 71-79.

Rogers, S.J., Hepburn, S.L., Stackhouse, T., \& Wehner, E. (2003). Imitation performance in toddlers with autism and those with other developmental disorders. Journal of Child Psychology and Psychiatry, 44, 763-781.

Sacks O. (1996). Un anthropologue sur Mars. Paris: Seuil.

Sauvage D. (1988). Autisme du nourrisson et du jeune enfant. Paris: Masson.

Schmitz C., Assaiante C., Gepner B. (2002). Modulation de la réponse anticipée en fonction du poids à délester : étude chez l'enfant sain et l'enfant autiste. Revue TIPA, 21, 207-211. 
Schmitz C., Martineau J., Barthélémy C. \& Assaiante C. (2003). Motor control and children with autism : deficit of anticipatory function? Neuroscience Letters, 348, 17-20.

Schultz, R.T., Gauthier, I., Klin, A., Fulbright, R., Anderson, A., Volkmar, F.R., Skudlarski, P., Lacadie, C., Cohen, D.J., \& Gore, J.C. (2000). Abnormal ventral temporal cortical activity during face discrimination among individuals with autism and Asperger syndrome. Archives of General Psychiatry, 57, 331-340.

Sellin, B. (1998). La solitude du déserteur. Paris : Robert Laffont.

Smith, I.M., \& Bryson, S.E. (1994). Imitation and action in autism: a critical review. Psychological Bulletin, $116,259-273$.

Spencer, J, O'Brien J, Riggs K, Braddick O, Atkinson J, Wattam-Bell J (2000). Motion processing in autism: evidence for a dorsal stream deficiency. NeuroReport, 11, 2765-2767.

Spencer, K.M., Nestor, P.G., Perlmutter, R., Niznikiewicz, M.A., Klump, M.C., Frumin, M., Shenton, M.E., McCarley, R.W. (2004). Neural synchrony indexes disordered perception and cognition in schizophrenia. Proceedings of the National Academy of Sciences,101, 7288-17293.

Stein, J. (2001) The magnocellular theory of developmental dyslexia. Dyslexia 7, 12-36.

Symond, M.P., Harris, A.W., Gordon, E., \& Williams, L.M. (2005). "Gamma synchrony" in first-episode schizophrenia: a disorder of temporal connectivity? American Journal of Psychiatry, 162, 459-465.

Takarae, Y., Minshew, N.J., Luna, B., Krisky, C.M., Sweeney, J.A. (2004). Pursuit eye movement deficits in autism. Brain, 127, 2584-94.

Tallal P. (1976). Rapid auditory processing in normal and disordered language development. Journal of Speech \& Hearing Research, 19, 561-594.

Tallal, P., Miller, S.L., Bedi, G., Byma, G., Wang, X., Nagarajan, S.S., Schreiner, C., Jenkins, W.M., Merzenich, M.M. (1996). Language comprehension in language-learning impaired children improved with acoustically modified speech. Science, 271, 81-83.

Talcott J.B., Hansen P.C., Assoku E.L., Stein J.F. (2000). Visual motion sensitivity in dyslexia: evidence for temporal and energy integration deficit. Neuropsychologia, 38, 935-943.

Tardif, C. et Gepner, B. (2003). L'Autisme. Collection 128, Nathan, Paris.

Tardif, C., Thomas, K., Gepner, B., Rey, V. (2002). Contribution à l'évaluation du système phonologique explicite chez des enfants autistes. Parole, 21, 35-72.

Tardif, C., Lainé, F., Rodriguez, M. \& Gepner, B. (2006, in press). Slowing down presentation of facial movements and vocal sounds enhances facial expression recognition and induces facial-vocal imitation in children with autism. Journal of Autism and Developmental Disorders.

Teitelbaum, P., Teitelbaum, O., Nye, J., Fryman, J. \& Maurer, R. (1998). Movement analysis in infancy may be useful for early diagnosis of autism. Proceedings of the National Academy of Sciences, 95, 13982-87.

Tekell, J.L., Hoffmann, R., Hendrickse, W., Greene, R.W., Rush, A.J., \& Armitage, R. (2005). High frequency EEG activity during sleep : characteristics in schizophrenia and depression. Clinical EEG and Neuroscience, 36, 25-35.

Tustin, F. (1972). Autisme et psychose de l'enfant. Trad. Française, Paris: Seuil, 1977.

Varela, F., Lachaux, J.P., Rodriguez, E. \& Martinerie, J. (2001). The Brainweb : phase synchronization and large-scale integration. Nature Reviews Neuroscience, 2, 229-239.

Vexiau, A-M. (1996). Je choisis ta main pour parler. Paris : Robert Laffont. 
Vilensky, J.A., Damasio, A.R., Maurer, R.G. (1981). Gait disturbances in patients with autistic behavior. A preliminary study. Archives of Neurology, 38, 646-649.

Villalobos, M.E., Mizuno, A., Dahl, B.C., Kemmotsu, N., \& Muller, R.A. (2005). Reduced functional connectivity between $\mathrm{V} 1$ and inferior frontal cortex associated with visuomotor performance in autism. Neuroimage, 15, 916-925.

Volkmar, F.R., Sparrow, S.S., Rende, R.C., \& Cohen, D.J. (1989). Facial perception in autism. Journal of Child Psychology and Psychiatry, 30, 591-598.

Volkmar, F. R., Pauls, D. (2003). Autism. Lancet, 362, 1133-41.

Waterhouse, L., Fein, D., \& Modahl, C. (1996). Neurofunctional mechanisms in autism. Psychological Review, $103,457-489$.

Welsh, J.P., Ahn, E.S., \& Placantonakis, D.G. (2005). Is autism due to brain desynchronization ? International Journal of Developmental Neuroscience, 23, 253-263.

Wickelgren, I. (2005). Autistic brains out of synch ? Science, 308, 1856-1858.

Wicker, B., Hubert, B., Fonlupt, P., Tardif, C., Gepner, B., \& Deruelle, C. (submitted). Abnormal effective connectivity during explicit emotional processing in autism.

Williams, D. (1992). Nobody nowhere, Doubleday.

Williams, J.H., Whiten, A., \& Singh, T. (2004). A systematic review of action imitation in autistic spectrum disorder. Journal of Autism and Developmental Disorders, 34, 285-299.

World Health Organization. (1992). The ICD-10 Classification of Mental and Behavioural Disorders (ICD-10), Geneva: WHO.

Zihl, J., Von Cramon, D., Mai, N. (1983). Selective disturbance of movement vision after bilateral brain damage. Brain, 106, 313-340.

Zwaigenbaum, L., Bryson, S., Rogers, T., Roberts, W., Brian, J., \& Szatmari, P. (2005). Behavioral manifestations of autism in the first year of life. International Journal of Developmental Neuroscience, 23, 143152. 
Figure 1. Schéma synthétique des cascades 'maldéveloppementales' secondaires à des désordres du traitement temporospatial des flux sensoriels dans l'autisme (repris de Gepner, 2005).

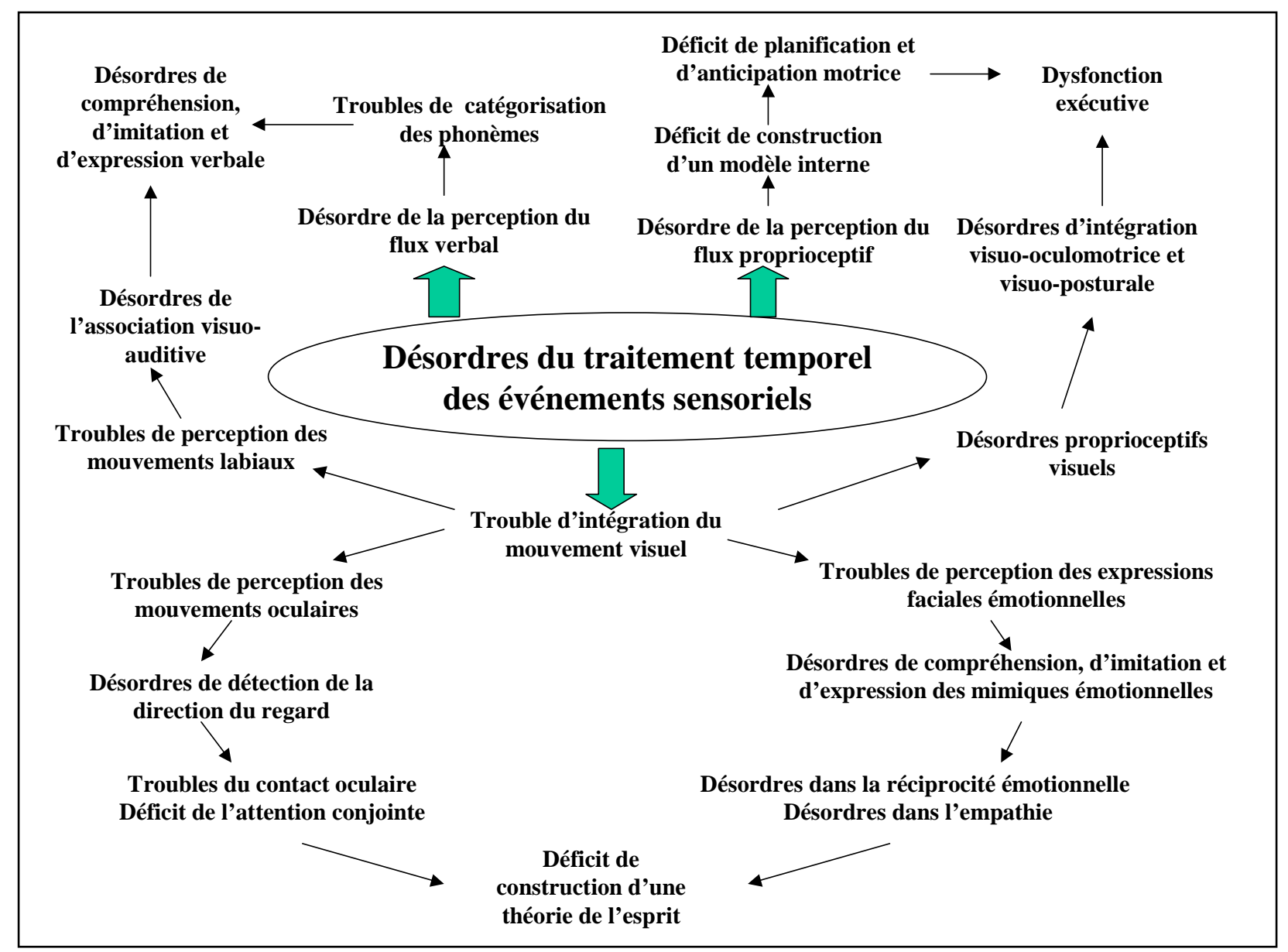

